\title{
ESTRATÉGIAS E MATERIAIS UTILIZADOS EM FOTOCATÁLISE HETEROGÊNEA PARA GERAÇÃO DE HIDROGÊNIO ATRAVÉS DA FOTÓLISE DA ÁGUA
}

Fabielle C. Marques ${ }^{\mathrm{a}}$, Alexandre M. Stumbo ${ }^{\mathrm{b}}$ e Maria C. Canela ${ }^{\mathrm{b}, *, \#}$

${ }^{a}$ Instituto Federal do Espírito Santo, Rodovia BR 482, Cachoeiro x Alegre - Km 6,5, Morro Grande, 29311-970 Cachoeiro de Itapemirim - ES, Brasil

'Laboratório de Ciências Químicas, Centro de Ciência e Tecnologia, Universidade Estadual do Norte Fluminense Darcy Ribeiro, Av. Alberto Lamego, 2000, 28013-602 Campos dos Goytacazes - RJ, Brasil

Recebido em 05/07/2016; aceito em 24/11/2016; publicado na web em 10/02/2017

\begin{abstract}
STRATEGIES AND MATERIALS USED IN HETEROGENEOUS PHOTOCATALYSIS APPLIED TO HYDROGEN GENERATION THROUGH WATER PHOTOLYSIS. Among the various technologies for the production of hydrogen fuel, heterogeneous photocatalysis is one of the most promising, especially with the use of semiconductors, notably $\mathrm{TiO}_{2}$. However, the use of $\mathrm{TiO}_{2}$ is limited by hindrances for the photolysis of water, such as wide bandgap, a less negative conduction band reduction potential as compared to that of hydrogen evolution and the high electron/hole recombination rate. Deactivation of the semiconductor can be avoided by the addition of electron-rich compounds (sacrificial reagents) which react irreversibly with the hole, leading to a higher quantum efficiency. Another strategy is the Z scheme. In this system, two different photocatalysts (or photosystems) are combined using a suitable redox mediator. Furthermore, the bandgap can be adjusted by doping with transition metal oxides, with control of metal oxide valence band using p-orbitals of an anion, or s-orbitals of p-block metal ions, or by spectral sensitization. In view of these questions, the purpose of this review article is to describe and discuss recent studies that use a variety of materials for the photocatalytic generation of hydrogen.
\end{abstract}

Keywords: hydrogen; photocatalysis; water splitting; Z scheme.

\section{INTRODUÇÃO}

A procura por fontes que assegurem o suprimento de energia, os altos preços do petróleo e as emissões crescentes de gases do efeito estufa constituem desafios ainda não resolvidos para a economia global e o clima do planeta. Há um consenso na comunidade internacional de que é preciso uma ação urgente para enfrentar os problemas de curto prazo sobre suprimento de energia e mitigar as mudanças futuras do clima, e que a tecnologia e a política são partes desta solução. ${ }^{1}$

Em 2015 foi publicado pela Agência Internacional de Energia (IEA), em Paris, o World Energy Outlook Special Report - Energy and Climate Change. Este relatório prevê um aumento da demanda por energia de $40 \%$ até 2030 , e a necessidade de redução das emissões e substituição de combustíveis fósseis. ${ }^{2}$ Com base nas projeções apresentadas pela IEA, pode-se esperar por um agravamento do aquecimento global e escassez de recursos fósseis se outras fontes de produção de energia não forem utilizadas. Portanto, a produção e utilização de energias alternativas tornam-se um dos maiores desafios da atualidade. De forma mais dramática, segundo o relatório Energy [R] evolution 2015, publicado pelo Greenpeace, Global Wind Energy Council e Solar Power Europe em setembro de 2015, a única maneira de estabilizar as emissões de $\mathrm{CO}_{2}$ até 2020 e reduzir a zero em 2050 seria alterar imediatamente a matriz energética mundial para $100 \%$ de energias renováveis. ${ }^{3}$

Dentre as fontes alternativas de energia, o hidrogênio é uma opção atrativa, pois possui elevada energia por unidade de massa (1 $\mathrm{kg}$ de hidrogênio contém aproximadamente a energia de $2,7 \mathrm{~kg}$ de gasolina), o que facilita a portabilidade da energia. ${ }^{4}$ Além disso, sua combustão não gera contaminante, mas apenas água.

*e-mail: mccanela@gmail.com

"e-mail alternativo: mccanela@uenf.br
Embora a combustão do hidrogênio não produza gases de efeito estufa, cerca de $95 \%$ do hidrogênio consumido no mundo é gerado a partir de materiais fósseis, sendo obtido principalmente a partir da reforma a vapor de hidrocarbonetos como o metano e nafta (subprodutos do refino do petróleo). ${ }^{5}$ Este processo, além de demandar grande quantidade de energia para ocorrer (a decomposição do combustível em hidrogênio somente ocorre em temperaturas superiores a $700{ }^{\circ} \mathrm{C}$ ), produz uma mistura de monóxido de carbono e hidrogênio. Para que a produção de hidrogênio seja considerada sustentável, é necessário o desenvolvimento de processos que atendam aos seguintes requisitos: que a fonte geradora seja renovável; que não haja grande demanda de energia; que não ocorra a produção de poluentes como subprodutos; e que seja um processo técnica e economicamente viável.

Os avanços tecnológicos nas áreas de produção de hidrogênio de forma eficiente, econômica e ambientalmente correta, estão acontecendo em todo o mundo. Dentre as diversas tecnologias para a produção de hidrogênio, destaca-se a de fotocatálise heterogênea, principalmente com uso de semicondutores. ${ }^{5-7}$ Apesar de ser um processo eficiente, o uso de luz UV artificial ainda torna o processo fotocatalítico pouco aplicável para escalas industriais. Na intenção de prover condições para a mudança neste paradigma, pesquisas recentes estão voltadas ao desenvolvimento de materiais com aproveitamento cada vez maior da luz visível do espectro solar, com o objetivo claro de se utilizar uma fonte de energia limpa, segura, renovável e principalmente abundante.

\section{PRINCÍPIOS DA FOTOCATÁLISE HETEROGÊNEA}

A fotocatálise heterogênea tem sua origem na década de setenta, quando pesquisas em células fotoeletroquímicas começaram a ser desenvolvidas, com o objetivo de produção de combustíveis a partir de materiais baratos, visando a transformação da energia solar em 
química. Em 1972, um trabalho de Fujishima e Honda ${ }^{8}$ descreveu a oxidação da água promovida por $\mathrm{TiO}_{2}$ em suspensão, o qual foi irradiado em uma célula fotoeletroquímica, gerando hidrogênio e oxigênio. A partir desta época, muitas pesquisas foram dedicadas ao entendimento de processos fotocatalíticos envolvendo a oxidação da água e íons inorgânicos, quando espécies semicondutoras eram irradiadas por luz. O desenvolvimento teórico que explica os fenômenos associados ao processo de desenvolvimento fotocatalítico dos semicondutores envolve os conceitos de teoria de bandas em sólidos e já foi descrita por vários autores. ${ }^{9-12}$

Os semicondutores de interesse em fotocatálise são sólidos em que os átomos constituem uma rede tridimensional infinita. A sobreposição dos orbitais atômicos vai além da vizinhança, estendendo-se por toda a rede ( $\mathrm{N}$ orbitais); resulta, então, numa configuração de estados deslocalizados muito próximos entre si, que formam a banda de valência (BV), de menor energia, e a banda de condução (BC), de maior energia. Entre as bandas há intervalos de energia nos quais não há estados eletrônicos "permitidos", sendo que cada um destes intervalos é chamado de banda de energia proibida ou gap. ${ }^{13}$

No caso da fotocatálise, a forma de gerar o par lacuna/elétron é via irradiação por luz com comprimento de onda dado por: $\lambda_{g}=E_{g} / h c$, em que $h$ é a constante de Planck e $c$ é a velocidade da luz. A absorção de fótons de energia maior que $E_{g}$ (fotoexcitação) promove elétrons da banda de valência para a banda de condução $\left(e_{B C}^{-}\right)$, e para cada elétron promovido, produz-se uma lacuna na banda de valência $\left(h^{+}{ }_{B V}\right)$. Como a maioria dos semicondutores é constituída por sólidos nanocristalinos, as cargas do par elétron/ lacuna podem migrar para a superfície da partícula e produzir sítios oxidantes e redutores.

As posições das bandas limites de vários semicondutores são apresentadas na Figura 1. A escala de energia interna é dada à esquerda por comparação com o nível no vácuo e à direita por comparação com um eletrodo normal de hidrogênio, $(\mathrm{ENH})$. As posições são derivadas dos potenciais das bandas dos sólidos em contato com uma solução de $\mathrm{pH}=1$. Enquanto os elétrons fotogerados na superfície podem ser doados para algum aceitador pré-adsorvido, as lacunas localizadas na $\mathrm{BV}$ podem mostrar potenciais bastante positivos (oxidantes), na faixa de $+0,5 \mathrm{~V}$ a $+3,0 \mathrm{~V}$, medidos contra um eletrodo normal de hidrogênio (ENH), e, portanto, receber elétrons com muita facilidade de espécies adsorvidas.

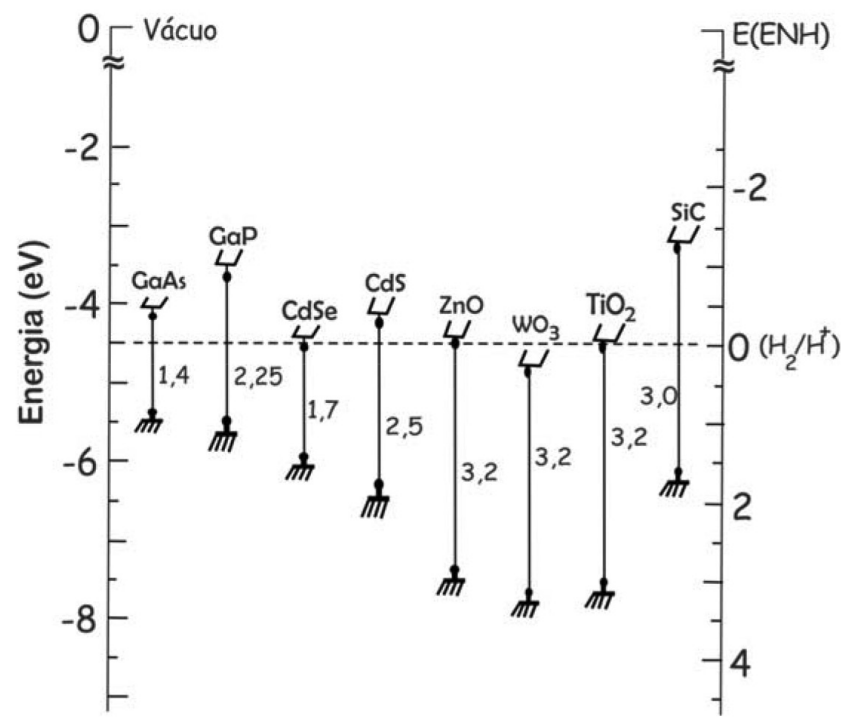

Figura 1. Principais semicondutores utilizados em fotocatálise: energia de bandgap e potencial redox em solução a pH=1. Reimpresso da Ref. $14 \mathrm{com}$ permissão. Copyright 1995 American Chemical Society

\section{GERAÇÃO DE $\mathrm{H}_{2}$ POR FOTOCATÁLISE}

A geração de $\mathrm{H}_{2}$ sem a utilização de um potencial externo, contrariando o método de Fujishima e Honda, é o que conhecemos hoje como Water Splitting, cuja teoria será explanada a seguir. Vários trabalhos insistem na utilização de semicondutores para produção de hidrogênio, notoriamente o $\mathrm{TiO}_{2}$, devido principalmente ao seu baixo custo, alta estabilidade, insolubilidade em água e baixa toxicidade..$^{15-17}$

Mesmo que o $\mathrm{TiO}_{2}$ possa ser fotoexcitado por uma irradiação acima do bandgap, a molécula de $\mathrm{H}_{2} \mathrm{O}$ não pode ser fotodecomposta sobre uma superfície de $\mathrm{TiO}_{2}$ devido à presença de um largo sobrepotencial para a evolução de $\mathrm{H}_{2}$ e $_{2}$ na superfície do $\mathrm{TiO}_{2}$. Sato e White demonstraram que a evolução de hidrogênio em umidade na superfície do $\mathrm{TiO}_{2}$ não é devido à quebra da molécula de água via fotocatálise, mas causada pela oxidação fotoassistida nas vacâncias de oxigênio de $\mathrm{TiO}_{2}$ reduzido. ${ }^{16}$

Os detalhes do mecanismo para a fotodecomposição da água no $\mathrm{TiO}_{2}$, ou reações de redução envolvendo o sistema $\mathrm{TiO}_{2}$-água, permanecem controversos até os dias atuais. Um número razoável de pesquisadores tem divulgado a formação de muitas espécies de radicais através de medidas de RPE. Gonzalez-Elipe et al. ${ }^{18}$ relataram a geração das espécies $\mathrm{HO}_{2}{ }^{\circ}$ e $\mathrm{O}^{-}$ou $\mathrm{O}_{2}{ }^{3 \cdot}$ por radiação de luz UV em $\mathrm{TiO}_{2}$ hidratado. Anpo et al. ${ }^{19}$ observaram a formação de radicais HO - a $77 \mathrm{~K} \mathrm{em}$ anatásio hidratado em condições de energia acima do bandgap. Radicais hidroxila não são produtos primários deste processo e sua existência é transiente, devido a sua alta reatividade. ${ }^{18}$ Este processo ocorre nas seguintes etapas (reações 1-4):

$$
\begin{gathered}
\text { fotocatalisador }+2 h \mathrm{~h} \rightarrow 2 \mathrm{e}^{-}+2 \mathrm{~h}^{+} \\
\mathrm{H}_{2} \mathrm{O} \rightarrow \mathrm{OH}^{-}+\mathrm{H}^{+} \\
2 \mathrm{e}^{-}+2 \mathrm{H}^{+} \rightarrow \mathrm{H}_{2} \\
2 h^{+}+2 \mathrm{OH}^{-} \rightarrow 2 \mathrm{H}^{+}+1 / 2 \mathrm{O}_{2}
\end{gathered}
$$

A reação total é:

$$
\begin{gathered}
\text { fotocatalisador }+\mathrm{H}_{2} \mathrm{O}+2 \mathrm{hv} \rightarrow \mathrm{H}_{2}+1 / 2 \mathrm{O}_{2} \\
\left(\text { razão molar } \mathrm{H}_{2} / \mathrm{O}_{2}=2\right)
\end{gathered}
$$

$\mathrm{Na}$ ausência de um catalisador, a reação global é termodinamicamente desfavorável $\left(\Delta \mathrm{G}^{\mathrm{o}}=+238 \mathrm{~kJ} \mathrm{~mol}^{-1}\right)$, e a Energia de Limiar $\left(\mathrm{E}_{\mathrm{L}}\right)$ necessária para a ocorrência da fotólise é dada pela Equação 1:

$$
E_{L}=\frac{\Delta G_{\left(\mathrm{H}_{2} \mathrm{O}\right)}^{0}}{2 N_{A}}
$$

Sendo que $N_{A}$ é o número de Avogadro. De acordo com a equação, a energia mínima para a fotodecomposição pode ocorrer com a incidência de energia luminosa com comprimentos de onda $\leq 1000$ $\mathrm{nm}$, ou seja, $>1,23 \mathrm{eV}$, o que corresponde a fótons com energia na faixa do infravermelho próximo. No entanto, para usar luz visível, o bandgap precisa ser $<3,0 \mathrm{eV}(>400 \mathrm{~nm}){ }^{20}$

Teoricamente, se o potencial de redução na banda de condução de um semicondutor for mais negativo do que aquele da evolução do hidrogênio, e se o potencial da banda de valência for mais positivo do que da evolução do oxigênio, o material possivelmente pode ser utilizado para decompor a água em $\mathrm{H}_{2}$ e em $\mathrm{O}_{2}$, conforme ilustrado na Figura 2.

Outra dificuldade na utilização do $\mathrm{TiO}_{2}$ é o baixo tempo de vida dos fotoelétrons gerados. O tempo de recombinação do par elétron/ lacuna é menor comparado ao tempo necessário para o par elétron/ lacuna alcançar a superfície. Assim, apenas uma pequena parte dos elétrons gerados consegue migrar para a superfície. ${ }^{15}$ Além da pouca quantidade de fotoelétrons na superfície, as velocidades das reações 


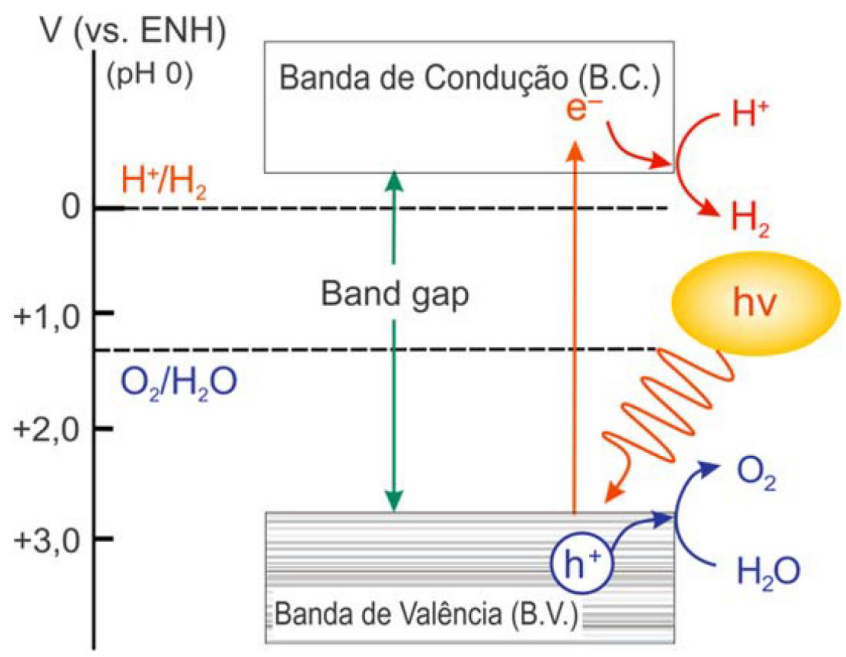

Figura 2. Princípio básico de geração fotocatalítica de $\mathrm{H}_{2}$ através da irradiação de semicondutores. Reimpresso da Ref. 21 com permissão. Copyright 2007 American Chemical Society

de redução e oxidação da água são menores comparadas à velocidade de recombinação do par elétron/lacuna. Conforme essas condições, a eficiência do $\mathrm{TiO}_{2}$ em água pura é baixíssima, tornando necessária a adição de agentes de sacrifício para viabilizar a utilização do $\mathrm{TiO}_{2}$ na produção de $\mathrm{H}_{2}$.

\section{Combinação: doadores de elétrons e fotocatalisador}

A razão dos baixos rendimentos de $\mathrm{H}_{2}$ quando se utiliza $\mathrm{TiO}_{2} \mathrm{em}$ água pura é a alta velocidade de recombinação elétron/lacuna que cessa o efeito fotocatalítico após curto período de iluminação. Uma das formas de se evitar a desativação do $\mathrm{TiO}_{2}$ é a aplicação de compostos doadores de elétrons (reagentes de sacrifício) para reagir de forma irreversível com as lacunas, resultando em uma maior eficiência quântica. Uma vez que o reagente de sacrifício é consumido durante a reação fotocatalítica, a adição contínua de doadores de elétrons é necessária para sustentar a produção de hidrogênio. ${ }^{17}$

Galinska e Walendziewski ${ }^{22}$ estudaram a influência de $\mathrm{I}^{-}, \mathrm{IO}_{3}{ }^{-}$, $\mathrm{Na}_{2} \mathrm{~S}$, EDTA e metanol na geração de $\mathrm{H}_{2}$ pela fotólise da água, usando o sistema $\mathrm{Pt}_{-} \mathrm{TiO}_{2}$ irradiado com luz UV, e verificaram que, na presença do metanol, a atividade da reação em função da geração de $\mathrm{H}_{2}$ foi a maior entre os doadores de elétrons utilizados (11,50 $\mathrm{mmol} / 90 \mathrm{~min}$ ), em função do hidrogênio ser parcialmente gerado pelo consumo do metanol. Contrapondo o trabalho de Hara et al.,$^{23}$ o EDTA, assim como o metanol, teve mais influência na geração de $\mathrm{H}_{2}$ do que o $\mathrm{Na}_{2} \mathrm{~S}$. A geração de $\mathrm{H}_{2}$ foi de $2,209 \mathrm{mmol} / 90 \mathrm{~min}$ quando se usou EDTA. Os estudos indicam que o EDTA captura radicais $\mathrm{HO}^{-}$e impede a recombinação do par elétron/lacuna, que resultaria na reação de oxigênio com hidrogênio. ${ }^{22}$

$\mathrm{O}$ uso de reagentes de sacrifício é uma alternativa para remover um dos produtos da fotodecomposição e assim deslocar o equilíbrio no sentido de aumentar a geração de hidrogênio. Neste caso, a espécie de sacrifício pode ser oxidada pelo produto da reação na lacuna (presumidamente $\mathrm{O}_{2}$ ) ou reduzida pelo produto originário pelo elétron fotogerado (presumidamente $\mathrm{H}_{2}$ ). ${ }^{17}$ Por exemplo, quando um álcool, tal como $\mathrm{CH}_{3} \mathrm{OH}$, é adicionado a uma suspensão aquosa de $\mathrm{TiO}_{2}$, sustenta-se que a produção de $\mathrm{H}_{2}$ é observada sob radiação UV e a molécula de álcool é oxidada a $\mathrm{CO}_{2} \cdot{ }^{14} \mathrm{Um}$ reforço a esta afirmação foi um estudo no qual uma grande variedade de componentes derivados de biomassa foi testada por Kondarides et al., ${ }^{24}$ a exemplo de monossacarídeos como pentoses (ribose, arabinose) e hexoses (glicose, galactose, frutose e manose), álcoois (metanol, etanol, propanol e butanol) e ácidos orgânicos (ácido acético, ácido fórmico). Em todos os casos, as quantidades de $\mathrm{H}_{2}$ e $\mathrm{CO}_{2}$ produzidas estão de acordo com a estequiometria da reação de reforma e todo o processo pode ser representado pela equação da reação global (6) abaixo:

$$
\mathrm{C}_{\mathrm{x}} \mathrm{H}_{\mathrm{y}} \mathrm{O}_{\mathrm{z}}+(2 \mathrm{x}-\mathrm{z}) \mathrm{H}_{2} \mathrm{O} \rightarrow \mathrm{x} \mathrm{CO}_{2}+(2 \mathrm{x}-\mathrm{z}+\mathrm{y} / 2) \mathrm{H}_{2}
$$

EDTA, metanol, etanol, $\mathrm{CN}^{-}$, ácido lático e formaldeído foram testados e revelaram-se eficazes para aumentar a produção de hidrogênio. ${ }^{25-27} \mathrm{Nada}$ et al. ${ }^{28}$ realizaram uma pesquisa de investigação qualitativa para estudar os efeitos de diferentes doadores de elétrons sobre a produção de hidrogênio. A classificação em termos do grau de capacidade de produção de hidrogênio foi: EDTA > metanol $>$ etanol > ácido lático. Deve-se notar que a decomposição destes hidrocarbonetos pode também contribuir para um rendimento mais elevado de hidrogênio, uma vez que o hidrogênio é um dos seus produtos de decomposição. ${ }^{28}$

Outra vertente em que os semicondutores têm sido aplicados como mediadores em processos fotocatalíticos é a de mineralização de poluentes orgânicos concomitante à geração de $\mathrm{H}_{2}$ em soluções aquosas. Jang et al..$^{29}$ estudaram a geração de hidrogênio simultaneamente à degradação fotocatalítica de $\mathrm{H}_{2} \mathrm{~S}$. Neste caso, os pesquisadores sintetizaram o compósito $\mathrm{CdS} / \mathrm{TiO}_{2}$ em suspensão aquosa e irradiaram com lâmpada de $\mathrm{Hg}$ de $500 \mathrm{~W}$ equipada com filtro para $\lambda \leq 420 \mathrm{~nm}$. O íon HS atuou com doador de elétrons, permitindo que a redução da água fosse intermitente.

A diversidade de reagentes de sacrifício testados por diversos autores é enorme, e podem ser escolhidos de acordo com as demandas locais. Preocupados com a poluição por resíduos provenientes da produção de óleo de oliva, pesquisadores italianos realizaram testes para geração de hidrogênio com concomitante degradação de moléculas orgânicas presentes no resíduo. Neste estudo, Speltini et $a l .{ }^{30}$ verificaram que, após $4 \mathrm{~h}$ de irradiação UV-A, uma suspensão a pH=3 de $2 \mathrm{~g} \mathrm{~L}^{-1} \mathrm{de} \mathrm{Pt} / \mathrm{TiO}_{2}$, contendo 3,3\% (v/v) de resíduo de produção de óleo de oliva produziu $44 \mu \mathrm{mol}$ de hidrogênio. Em outro trabalho do mesmo autor, hidrogênio foi produzido por fotocatálise via water splitting na presença de esgoto proveniente de suinocultura como reagente de sacrifício. No trabalho, o hidrogênio foi gerado sob irradiação UV-A e luz solar na presença de óxido de titânio platinizado como catalisador. O resíduo foi coletado num estabelecimento localizado no norte da Itália e, após centrifugado e diluído com água, foi testado para a geração de $\mathrm{H}_{2}$ na presença do catalisador e luz. Sob condições ótimas, os autores obtiveram $50 \mu \mathrm{mol}$ de gás hidrogênio em $4 \mathrm{~h}$ de teste fotocatalítico, no qual se utilizou 1,7\% em volume do resíduo. No estudo, fez-se uma comparação com outros reagentes de sacrifício usuais e o rendimento em termos de hidrogênio foi aproximadamente o dobro em comparação com o esgoto de suínos, indicando assim a viabilidade da concomitante geração de $\mathrm{H}_{2}$ com eliminação de poluentes. ${ }^{31}$ Neste mesmo propósito, Souza e Silva ${ }^{32}$ avaliaram a geração fotocatalítica de hidrogênio usando lodo de curtume como reagente de sacrifício. No estudo, os pesquisadores utilizaram CdS como fotocatalisador e Pt como co-catalisador e os testes foram realizados sob luz visível. Em condições ótimas ( $\mathrm{pH}=13$, $60 \mathrm{mg} \mathrm{CdS}, 50 \%$ de resíduo e ausência de Pt), a quantidade de hidrogênio gerada foi de $184 \mu \mathrm{mol}$ após 27 h de irradiação. Ainda, verificou-se que a concentração de íons $\mathrm{S}^{-2}$ (presente no processo de produção do resíduo) diminuiu durante o teste devido à sua atuação como capturador de lacunas fotogeradas.

A degradação de corantes acompanhada pela geração de hidrogênio também foi explorada por Ravishankar et al. ${ }^{33}$ No estudo, nanopartículas de $\mathrm{TiO}_{2}: \mathrm{Ag}$ foram utilizadas num teste fotocatalítico em situação real, ou seja, aproveitando a luz solar $\left(750 \mathrm{~W} \mathrm{~m}^{-2}\right)$ dos meses de novembro a dezembro em Bangalore, India. Testes também 
foram realizados em situação simulada, utilizando-se luz ultravioleta $\left(125 \mathrm{~W} \mathrm{~m}^{-2}\right)$. O corante utilizado como reagente de sacrifício foi o trypan blue, material muito utilizado para tingimento de náilon, lã, algodão, seda e também para a coloração de óleos, gorduras, ceras, vernizes, plásticos, etc. Os autores concluiram que as nanopartículas $\mathrm{TiO}_{2}$ :Ag geraram $2230 \mu \mathrm{mol}$ de $\mathrm{H}_{2}$ por $1 \mathrm{~g}$ de fotocatalisador, durante $2,5 \mathrm{~h}$ de teste. A degradação total do corante se processou em concentrações menores do poluente (5-15 ppm) e tendeu a diminir a conversão a concentrações maiores, atribuída à saturação de sítios ativos e diminuição da penetração de luz.

Alguns trabalhos utilizaram com sucesso o glicerol para reforma via fotocatálise, com concomitante geração de $\mathrm{H}_{2}$ pela decomposição fotocatalítica da água. Daskalaki e Kondarides ${ }^{34}$ investigaram a fotorreforma de glicerol com o uso do fotocatalisador $\mathrm{Pt} / \mathrm{TiO}_{2}$ por irradiação de luz ultravioleta. Os resultados obtidos mostram que o hidrogênio pode ser produzido eficientemente a partir da reforma fotocatalítica de soluções aquosas de glicerol e, eventualmente, uma conversão completa de glicerol a $\mathrm{H}_{2} \mathrm{e} \mathrm{CO}_{2}$. Os melhores resultados foram obtidos com o fotocatalisador $\mathrm{TiO}_{2}$ carregado com $0,1-2,0 \%$ em massa de $\mathrm{Pt}$, proporcionando taxas de produção de hidrogênio de 24,0 a $28,2 \mu \mathrm{mol} \mathrm{h}{ }^{-1}$. De maneira similar, Liu et al. ${ }^{35}$ produziram hidrogênio por fotocatálise utilizando $\mathrm{TiO}_{2}$ impregnado com $10 \%$ em massa de $\mathrm{NiO}_{x}$ numa suspensão de água e glicerol a $20 \%$ (v/v). $\mathrm{O}$ teste foi realizado utilizando uma lâmpada de vapor de mercúrio de $500 \mathrm{~W}$, cuja radiação se encontra predominantemente em $365 \mathrm{~nm}$, 460 nm e 466 nm. A velocidade inicial para produção de hidrogênio com concomitante reforma do glicerol foi de $900 \mu \mathrm{mol} \mathrm{h}^{-1}$. Languer et al..$^{36}$ também investigaram a fotorreforma do glicerol, utilizando nanotubos de $\mathrm{TiO}_{2}$ impregnados com Pt. Os nanotubos foram preparados pela anodização de Ti, e após tratamento térmico, Pt foi depositada no material. Sob irradiação UV, os autores do trabalho observaram a formação de $0,2 \mu \mathrm{mol} \mathrm{h} \mathrm{h}^{-1} \mathrm{~cm}^{-2}$ de hidrogênio numa suspensão contendo o fotocatalisador numa solução de água/glicerol na proporção 1/6,6 (v/v).

Num trabalho semelhante ao de Liu et al., ${ }^{35}$ Fujita et al. ${ }^{37}$ realizaram a reação de fotólise da água com a utilização de $\mathrm{NiO} / \mathrm{TiO}_{2}$ suspenso numa solução aquosa de glicerol, irradiado com lâmpada de vapor de mercúrio de $500 \mathrm{~W}$. No estudo, os pesquisadores verificaram a influência da concentração do glicerol na velocidade de geração de hidrogênio. A velocidade de geração de $\mathrm{H}_{2}$ aumentou linearmente com a concentração de glicerol, quando este foi menor que $0,5 \mathrm{~mol} \mathrm{~L}^{-1}$. Acima disso, a velocidade de reação não aumentou mais que 1200 $\mu \mathrm{mol} \mathrm{g}^{-1} \mathrm{~h}^{-1}$. Isto indicou, segundo os autores, que a interação do glicerol no catalisador obedece o modelo de adsorção de Langmuir. A constante da velocidade de adsorção de pseudo-primeira ordem foi de $1,3 \mathrm{~mol} \mathrm{~g}^{-1} \mathrm{~h}^{-1} \mathrm{e}$ a constante de equilíbrio para adsorção-dessorção de glicerol foi de 3,0 $\mathrm{L} \mathrm{mol}^{-1}$.

\section{Novos materiais empregando o Esquema Z}

O sistema convencional de geração fotocatalítica de $\mathrm{H}_{2}$, descrita na Figura 2, em que hidrogênio e oxigênio gasosos são formados em um único passo, apresenta algumas dificuldades experimentais:

a) muitos materiais só são ativos na região do UV;

b) mesmo absorvendo luz visível, apresentam um potencial de redução da banda de condução mais positivo que o potencial de redução do $\mathrm{H}^{+}$a $\mathrm{H}_{2}$;

c) também requerem o uso de reagentes de sacrifício como doares irreversíveis de elétrons para evitar a recombinação elétron/ lacuna. Neste caso, a geração de $\mathrm{H}_{2}$ não segue o mecanismo convencional;

d) a maioria dos fotocatalisadores não gera $\mathrm{H}_{2}$ e $\mathrm{O}_{2}$ em proporções estequiométricas (2:1).
O desenvolvimento de fotocatalisadores irradiados por luz visível para decomposição da água em duas etapas foi originalmente introduzido por Bard, ${ }^{38}$ em 1979. Este sistema, inspirado na fotossíntese natural em plantas verdes, em que $\mathrm{CO}_{2}$ e água são combinados para formar carboidrato e $\mathrm{O}_{2}$, foi chamado Esquema Z. Neste sistema, dois diferentes fotocatalisadores (ou fotossistemas) são combinados usando um mediador redox apropriado. Neste caso, a luz visível pode ser utilizada de forma mais eficiente do que em sistemas convencionais, porque a energia necessária para conduzir cada fotocatalisador é reduzida.

A estratégia é utilizar materiais ativos no visível, porém em uma combinação de tal forma que um dos fotocatalisadores tenha um potencial de redução na banda de condução mais negativo que o potencial de redução do $\mathrm{H}^{+}$a $\mathrm{H}_{2}$ e outro tenha um potencial na banda de valência mais positivo que o potencial de oxidação da água em $\mathrm{O}_{2}$. Por exemplo, o potencial da banda de condução do $\mathrm{WO}_{3}$ impede a sua utilização para uma evolução de $\mathrm{H}_{2}$, mesmo na presença de metanol. ${ }^{39}$ No entanto, $\mathrm{WO}_{3}$ é capaz de produzir $\mathrm{O}_{2}$ de uma solução aquosa contendo recebedores de elétrons apropriados $\left(\mathrm{Fe}^{2+} / \mathrm{Fe}^{3+}\right)$ sob luz visível, conforme ilustrado na Figura $3 .^{40}$

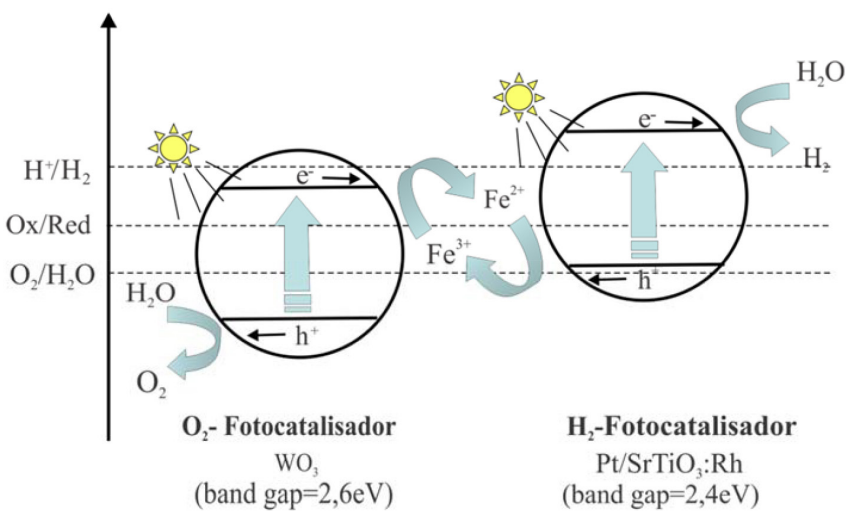

Figura 3. Exemplo do Esquema Z. Reimpresso da Ref. 40 com permissão. Copyright 2010 Elsevier Inc.

No caso acima, as reações envolvidas são:

Fotossistema 1 (reações 7-10):

$$
\begin{gathered}
2 \mathrm{H}_{2} \mathrm{O} \rightarrow 2 \mathrm{OH}^{-}+2 \mathrm{H}^{+} \\
\text {fotocatalisador }+2 h \mathrm{~h} \rightarrow 2 \mathrm{e}^{-}+2 \mathrm{~h}^{+} \\
2 \mathrm{e}^{-}+2 \mathrm{H}^{+} \rightarrow \mathrm{H}_{2} \\
2 \mathrm{Fe}^{2+}+2 h^{+} \rightarrow 2 \mathrm{Fe}^{3+}+2 \mathrm{e}^{-}
\end{gathered}
$$

A reação de oxidação do ferro acima ocorre na banda de valência do semicondutor, que apresenta potencial para reduzir a água. A reação de redução do $\mathrm{Fe}^{3+}$, que ocorre na banda de condução do material com a capacidade de evoluir o $\mathrm{O}_{2}$, seria:

Fotossistema 2 (reações 7,8, 11,12):

$$
\begin{gathered}
2 \mathrm{H}_{2} \mathrm{O} \rightarrow 2 \mathrm{OH}^{-}+2 \mathrm{H}^{+} \\
\text {fotocatalisador }+2 h \mathrm{~h} \rightarrow 2 \mathrm{e}^{-}+2 \mathrm{~h}^{+} \\
2 h^{+}+2 \mathrm{OH}^{-} \rightarrow 2 \mathrm{H}^{+}+1 / 2 \mathrm{O}_{2} \\
2 \mathrm{Fe}^{3+}+2 \mathrm{e}^{-} \rightarrow 2 \mathrm{Fe}^{2+}
\end{gathered}
$$

As vantagens deste sistema são:

a) Quando o potencial redox do mediador está localizado entre os potenciais de $\mathrm{H}^{+} / \mathrm{H}_{2} \mathrm{O}$ e $\mathrm{O}_{2} / \mathrm{H}_{2} \mathrm{O}$, a mudança de potencial químico $(\Delta \mathrm{G})$ em cada fotossistema é menor do que a reação total;

b) É possível dividir os fotocalisadores em dois vasos reacionais, separando-os por membranas, e, neste caso, observa-se a evolução dos gases separadamente; 
c) Dispensa a utilização de reagentes de sacrifício. No Esquema Z, os mediadores atuam de forma reversível.

As desvantagens deste sistema são: primeiro, que deve-se utilizar o dobro de fótons, quando comparado com o sistema convencional, apesar de os comprimentos de onda utilizados nos dois fotossistemas serem diferentes. E segundo, requer, obviamente, dois fotocatalisadores, que podem dificultar a operação do sistema por suas diferentes características, como, por exemplo, desativação. ${ }^{41}$

Um exemplo de utilização do Esquema $\mathrm{Z}$ foi reportado por Higashi et al.,${ }^{42}$ no qual os pesquisadores utilizaram oxinitretos mistos de tântalo $\mathrm{ATaO}_{2} \mathrm{~N}(\mathrm{~A}=\mathrm{Ca}, \mathrm{Sr}, \mathrm{Ba})$ para geração de hidrogênio. Os pesquisadores utilizaram a combinação $\mathrm{Pt} / \mathrm{ATaO}_{2} \mathrm{~N}$ e $\mathrm{Pt} / \mathrm{WO}_{3}$ em água, na presença dos mediadores redox $\mathrm{I}^{-} / \mathrm{IO}_{3}{ }^{-}$. Demonstrou-se que a presença do par redox melhora a atividade na região visível, para produção estequiométrica de $\mathrm{H}_{2}$ e $\mathrm{O}_{2}$ pela fotólise da água, e o fotocalisador manteve-se estável quanto a fotodecomposição a $\mathrm{N}_{2}$. A evolução da produção de hidrogênio e oxigênio na superfície do Pt/ $\mathrm{ATaO}_{2} \mathrm{~N}$ e Pt/ $/ \mathrm{WO}_{3}$, respectivamente, é mostrada na Figura 4:

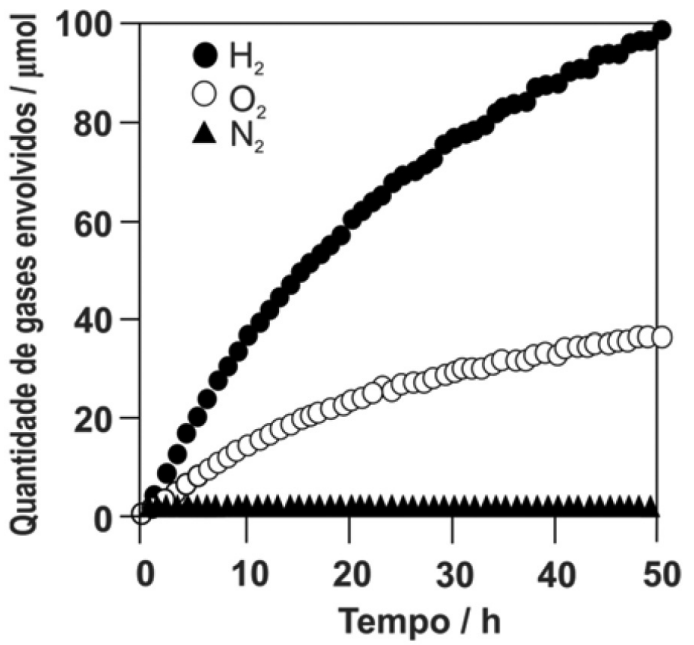

Figura 4. Evolução no tempo para o $\mathrm{H}_{2}$ e $\mathrm{O}_{2}$ sobre a mistura de do Pt/BaTa$\mathrm{O}_{2} \mathrm{~N}(30 \mathrm{mg})$ e $\mathrm{Pt}$ - $\mathrm{WO}_{3}(30 \mathrm{mg})$ em solução aquosa de $5 \mathrm{mmol} / \mathrm{L}$ de $\mathrm{NaI}$ sob luz visivel $(\lambda>420 \mathrm{~nm})$. Reimpresso da Ref. 42 com permissão. Copyright 2009 American Chemical Society

Em outro trabalho, Yan et al. ${ }^{43}$ utilizaram a combinação de $\mathrm{TiO}_{2}$ com $\mathrm{C}_{3} \mathrm{~N}_{4}$ para a reação de fotólise da água. $\mathrm{O}$ material foi suspenso numa solução de mediadores redox $\mathrm{I}^{-} / \mathrm{IO}_{3}^{-}$ou $\mathrm{Fe}^{2+/} \mathrm{Fe}^{3+}$ e irradiado com lâmpada de xenônio (comprimento de onda variando de 200 a 2500 $\mathrm{nm}$ ). Os autores relataram que o material apresentou boa estabilidade, e a velocidade de reação em função da evolução de hidrogênio foi de $154 \mu \mathrm{mol} \mathrm{h}^{-1}$.

\section{Outras estratégias e materiais usados em fotocatálise na geração de $\mathrm{H}_{2}$}

Decompor a água utilizando um óxido metálico sob luz visível $(\lambda>400 \mathrm{~nm})$ permanece um desafio. Em geral, o potencial da banda de condução de um fotocatalisador de óxido metálico é formado pelos orbitais $d$ vazios de um metal de transição ou orbitais $s, p$ de um típico metal, que se encontra acima do potencial de redução do $\mathrm{H}^{+}$a $\mathrm{H}_{2}(0$ $\mathrm{V} v s \mathrm{ENH}$ a $\mathrm{pH}$ 0). Por outro lado, o potencial da banda de valência, que consiste de orbitais $2 p$ do oxigênio, é consideravelmente mais positivo do que o potencial de oxidação da água $(1,23 \mathrm{~V}$ vs ENH a pH 0). Scaife ${ }^{44}$ estudou esta situação, examinando a relação entre o potencial de flat-band $\left(V_{F B}\right)$ e o bandgap $\left(E_{g}\right)$ de vários óxidos metálicos, encontrando a seguinte Equação 2 empírica:

$$
V_{F B}(E N H) \approx 2,94-E_{g}
$$

A banda de condução de semicondutores do tipo-n é quase sempre o mesmo que o potencial de flat-band, e a energia de bandgap de semicondutores para se utilizar a luz visível dever ser menor que $3 \mathrm{eV}$. Assim, o potencial da banda de condução para muitos óxidos semicondutores que são ativos no visível deveria ser positivo, tornando inviável a redução da água a $\mathrm{H}_{2}$. No entanto, a equação empírica, relatada por Scaife, não é obedecida para alguns óxidos que contêm metais de transição, tais como $\mathrm{Cr}^{3+}, \mathrm{Ni}^{2+}$ e $\mathrm{Fe}^{3+} .{ }^{45}$

Do ponto de vista da conversão de energia solar, no entanto, o desenvolvimento de um fotocatalisador que decompõe a água de forma eficiente em luz visível ( $\lambda>400 \mathrm{~nm}$ ) é indispensável. Até o início dos anos 1990, apenas alguns calcogenetos e óxidos metálicos (por exemplo, o $\mathrm{CdS}$ e $\mathrm{WO}_{3}$ ) eram conhecidos por serem fotocataliticamente ativos sob luz visível. ${ }^{45-47}$ Certos calcogenetos metálicos, incluindo $\mathrm{CdS}$ e $\mathrm{CdSe}$, pareciam ser fotocatalisadores adequados para decomposição da água, exibindo energias de bandgap suficientemente pequenas para permitir absorção de luz visível e ter potenciais da banda de condução e de valência apropriados para a redução da água e para a oxidação. Estes calcogenetos metálicos, no entanto, não são estáveis, porque os anions $\mathrm{S}^{2-} \mathrm{e} \mathrm{Se}^{2-}$ são mais suscetíveis à oxidação do que a água, fazendo com que os catalisadores $\mathrm{CdS}$ ou CdS e sejam oxidados e degradados. Ainda assim, recentes trabalhos sugerem que o ajuste principalmente de $\mathrm{pH}$ e salinidade do meio podem aumentar a estabilidade de CdS por reduzir a solubilidade do sulfeto em solução aquosa, ou mesmo que esta estabilidade pode ser melhorada com a utilização de sulfetos metálicos em sistemas fotocatalíticos multifases, como por exemplo, $\mathrm{Cd}_{1-\mathrm{x}} \mathrm{Zn}_{\mathrm{x}} \mathrm{S} / \mathrm{Zn}(\mathrm{OH})_{2}{ }^{48-51}$

Apesar do $\mathrm{WO}_{3}$ funcionar como um fotocatalisador estável para a evolução de $\mathrm{O}_{2}$ sob luz visível, na presença de um aceitador de elétrons apropriado, a banda de condução do material é mais alta do que o potencial de redução de água e, como resultado, não ocorre a redução do $\mathrm{H}^{+}$ $\mathrm{a} \mathrm{H}_{2}$. A Figura 5 mostra uma ilustração esquemática das estruturas de banda de alguns fotocatalisadores, destacando o dilema acima referido.

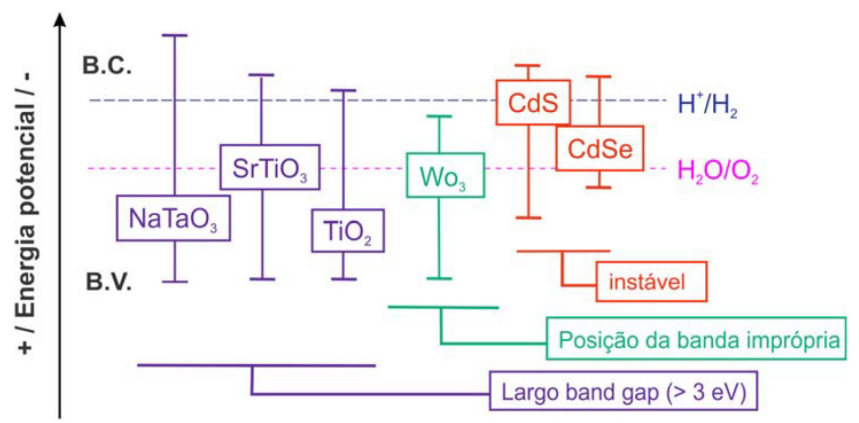

Figura 5. Ilustração esquemática das estruturas de banda de vários fotocatalisadores semicondutores e suas limitações. Reimpresso da Ref. $21 \mathrm{com}$ permissão. Copyright 2007 American Chemical Society

De acordo com Maeda, ${ }^{39}$ a dificuldade no desenvolvimento de um fotocatalisador adequado pode ser atribuído à falta de materiais conhecidos que satisfaçam a três requisitos:

1 Potenciais adequados para a decomposição da água

2 Energia de bandgap inferior a $3 \mathrm{eV}$

3 Estabilidade do fotocatalisador

As possíveis saídas sugeridas para estes problemas podem ser:

a) A dopagem de óxidos com íons de metais de transição com configuração eletrônica do tipo $d^{n}(0<n<10)$

b) controle da banda de valência de óxidos metálicos usando orbitais $p$ de um ânion ou os orbitais s de íons de metal do bloco $p$

c) sensibilização espectral 
A maioria dos óxidos metálicos que são ativos no visível apresentam potencial da banda de condução um pouco abaixo de $0 \mathrm{~V}$ em $\mathrm{pH}=0$ e potencial da banda de valência acima de $3 \mathrm{~V}$. Isto faz com que apresentem um bandgap muito grande para absorção de luz visível, mas sugere que fotocatalisadores baseados em óxidos metálicos tenham potencial suficiente para oxidar a molécula de água por conta da diferença entre o potencial de oxidação de $\mathrm{H}_{2} \mathrm{O}$ a $\mathrm{O}_{2}(1,23 \mathrm{~V} v s \mathrm{NHE})$ e a banda de valência (cerca de $3 \mathrm{~V}$ vs NHE). Por isso é importante modificar um óxido para absorção no visível, porém, mantendo o potencial da banda de condução.

Alguns trabalhos mostraram a potencialidade da fotocatálise heterogênea visando a produção de hidrogênio utilizando as sugestões acima. Sato et $a l .^{52}$ verificaram a decomposição de água ausente de impurezas em $\mathrm{H}_{2}$ e $\mathrm{O}_{2}$ sobre catalisadores de $\mathrm{RuO}_{2}$ impregnado com $\mathrm{MIn}_{2} \mathrm{O}_{4}(\mathrm{M}=\mathrm{Ca}, \mathrm{Sr}, \mathrm{Ba}), \mathrm{Sr}_{2} \mathrm{SnO}_{4}$ e $\mathrm{NaSbO}_{3}$ e irradiados por lâmpada de Xe. Estas combinações de óxidos com íons metálicos do bloco $p$ com configuração $d^{10}\left(\mathrm{In}^{3+}, \mathrm{Sn}^{4+} \mathrm{e} \mathrm{Sb}^{5+}\right)$ revelaram-se inovadores em relação aos fotocatalisadores convencionais que utilizam metais com coordenação octaédrica $\left(d^{0}\right)$, tal como $\mathrm{Ti}^{4+}, \mathrm{Zr}^{4+}, \mathrm{Nb}^{5+} \mathrm{e} \mathrm{Ta}^{5+}$.

Ye et al..$^{53}$ estudaram a geração de $\mathrm{H}_{2}$ pela decomposição da água utilizando o vanadato de índio $\left(\mathrm{InVO}_{4}\right)$ impregnado com óxido de níquel em suspensão de água pura e irradiado com luz visível, usando uma lâmpada de xenônio de $400 \mathrm{~W}$ com filtro para $\lambda<420 \mathrm{~nm}$. A velocidade de formação de $\mathrm{H}_{2}$ sob as condições descritas acima foi de 5,0 mmol g-1 $\mathrm{h}^{-1}$. A boa atividade fotocatalítica do material foi atribuída ao baixo bandgap ( $2 \mathrm{eV}$ ), estimado por espectroscopia UVVis, e indicação de diminuição do potencial da banda de condução.

Contrapondo as ideias de Sato et al. ${ }^{52}$, Iwase et al. ${ }^{54}$ insistiram com o sistema de óxido misto, $\mathrm{K}_{4} \mathrm{Nb}_{6} \mathrm{O}_{17}, \mathrm{Sr}_{2} \mathrm{Nb}_{2} \mathrm{O}_{7}, \mathrm{KTaO}_{3}, \mathrm{NaTaO}_{3}$, porém, realizando a foto deposição de $\mathrm{Au}$ sobre a superfície desses materiais. A evolução de $\mathrm{O}_{2}$ e $\mathrm{H}_{2}$ pela decomposição da água foi avaliada irradiando a partícula do catalisador com uma lâmpada de $\mathrm{Xe}$ de $300 \mathrm{~W}$. Neste caso, o Au atuou como eficiente co-catalisador e os resultados mostraram que quando se utilizou $\mathrm{NaTaO}_{3}: \mathrm{La}$ na ausência de $\mathrm{Au}$, a atividade em termos de $\mathrm{H}_{2}$ foi de $191 \mu \mathrm{mol} \mathrm{h} \mathrm{h}^{-1}$, porém, na presença de $\mathrm{Au}(0,3 \%$ em massa), a atividade foi elevada para $472 \mu \mathrm{mol} \mathrm{h}{ }^{-1}$.

Zou et al. ${ }^{55}$ estudaram a evolução de $\mathrm{H}_{2}$ de uma suspensão de água pura irradiada por luz visível $(\lambda>420 \mathrm{~nm})$ usando $\mathrm{NiO}_{x} / \mathrm{InTaO}_{4}$ e $\mathrm{NiO}_{\mathrm{x}} / \mathrm{InNbO}_{4}$ e verificaram que a atividade foi $4,0 \mu \mathrm{mol} \mathrm{g} \mathrm{g}^{-1} \mathrm{~h}^{-1} \mathrm{e}$ $3,5 \mu \mathrm{mol} \mathrm{g} \mathrm{g}^{-1} \mathrm{~h}^{-1}$, respectivamente. A atividade fotocatalítica na região do visível foi atribuída ao bandgap dos materiais, que foi calculado em 2,6 eV para o $\mathrm{InTaO}_{4}$ e 2,5 eV para o $\mathrm{InNbO}_{4}$. A diferença de atividade dos dois materiais foi relacionada ao potencial da banda de condução. No caso do $\mathrm{InNbO}_{4}$, a banda encontra-se no orbital $4 d$, enquanto que a do $\mathrm{InTaO}_{4}$ está no $5 d$. Estes potenciais das bandas dos fotocatalisadores indicam que o potencial da banda de condução do $\mathrm{InTaO}_{4}$ é mais negativo, o que favorece a evolução do $\mathrm{H}_{2}$.

A dopagem no $\mathrm{TiO}_{2}$ por metais de transição, como sugerido acima, também se mostra como alternativa para o incremento da atividade fotocatalítica do óxido frente à irradiação visível. Dholam et al. ${ }^{56}$ fizeram um estudo no qual filmes de $\mathrm{TiO}_{2}$ eram dopados com $\mathrm{Cr}$ e $\mathrm{Fe}$ e, posteriormente, foi verificada a atividade frente a geração de hidrogênio. Os materiais após a síntese eram testados como fotoanodos, mergulhados numa mistura água+eletrólito e iluminados com lâmpada de tungstênio de $250 \mathrm{~W}$, num reator de vidro borosilicato, que era utilizado como filtro de UV.

A geração de hidrogênio no $\mathrm{TiO}_{2}$ dopado com $\mathrm{Fe}^{+3}$ foi maior que no dopado com $\mathrm{Cr}^{+3}$, devido à habilidade dos íons ferro em trapear ambos elétron e lacuna e evitar a recombinação. Além disso, os resultados de DRS-UV-Vis mostraram boa absorção dos materiais na região do visível.

Muitos trabalhos envolvendo a geração fotocatalítica de hidrogênio estão baseados principalmente na utilização de óxidos, sulfetos e oxinitretos semicondutores, como alguns já citados acima. Há de se considerar a utilização recente de materiais sustentáveis, livres de metais, e com boa atividade no visível. Cita-se, por exemplo, o g- $\mathrm{C}_{3} \mathrm{~N}_{4}$ (nitreto de carbono grafítico), um polímero semicondutor que tem se mostrado um bom fotocatalisador para geração de $\mathrm{H}_{2} \mathrm{ou}$ $\mathrm{O}_{2}$ via water splitting sob irradiação de luz visível. No entanto, o uso de g- $\mathrm{C}_{3} \mathrm{~N}_{4}$ sozinho é limitado devido à alta recombinação do par lacuna-eletrón. Xiang et al. ${ }^{57}$ prepararam um compósito de grafeno/ $\mathrm{C}_{3} \mathrm{~N}_{4}$ e testaram a atividade para a geração fotocatalítica de hidrogênio no visível. Os autores verificaram que a condição ótima de $1,0 \%$ em massa de grafeno impregnado no $g-\mathrm{C}_{3} \mathrm{~N}_{4}$ houve a geração de $\mathrm{H}_{2}$ a uma velocidade de $451 \mu \mathrm{mol} \mathrm{h}{ }^{-1}$, sendo aproximadamente 3 vezes superior à atividade do $\mathrm{g}-\mathrm{C}_{3} \mathrm{~N}_{4}$ puro. A melhora no desempenho do material se deveu, segundo os autores, à presença do grafeno, que atuou como aceitador de elétrons fotogerados e, consequentemente, a recombinação do par lacuna-elétron foi evitada. Lan et al. ${ }^{58}$ também utilizaram o g- $\mathrm{C}_{3} \mathrm{~N}_{4}$, porém, dopado com $\mathrm{Br}$ para geração de hidrogênio por fotólise da água. Neste trabalho, $50 \mathrm{mg}$ do material foi adicionado a $100 \mathrm{~mL}$ de solução aquosa contento $10 \%$ em volume de trietanolamina como reagente de sacrifício. Ainda, em solução, a superfície do polímero foi impregnada com 3\% em massa de Pt por fotodeposição utilizando $\mathrm{H}_{2} \mathrm{PtCl}_{6}$. A suspensão foi iluminada com uma lâmpada de xenônio equipada com filtro para eliminar radiação UV. A velocidade de reação determinada para o $\mathrm{H}_{2}$ foi de $20 \mu \mathrm{mol}$ $\mathrm{h}^{-1}$ quando se utilizou polímero puro e $48 \mu \mathrm{mol} \mathrm{h} \mathrm{h}^{-1}$ com o polímero dopado numa quantidade otimizada de $\mathrm{Br}$. $\mathrm{O}$ trabalho indicou que a melhora da atividade do polímero com a presença do heteroátomo se deveu à formação de um nível de energia acima da banda de valência do semicondutor, diminuindo assim o bandgap.

Outra estratégia investigada é a heterojunção de semicondutores. Em contraste com o uso de fotocatalisadores individuais, a heterojunção de semicondutores forma um sistema eficiente para minimizar a recombinação elétron-lacuna. Diferentemente do Esquema Z, em que é necessária a presença de um mediador redox na solução aquosa para retardar a recombinação dos portadores de carga, na heterojunção de semicondutores, elétrons ou lacunas podem migrar de um material para outro, na ausência de um mediador. No último caso, os semicondutores devem estar em contato.

Yaojun et al. ${ }^{59}$ avaliaram a geração fotocatalítica de hidrogênio utilizando nanocompositos CdS/titanotubos em suspensão aquosa (utilizando $\mathrm{Na}_{2} \mathrm{~S}$ e $\mathrm{Na}_{2} \mathrm{SO}_{3}$ ) irradiada com uma lâmpada de xenônio equipada com filtro para eliminar radiação UV. Os resultados mostraram que o compósito exibiu excelente atividade no visível, com geração de $\mathrm{H}_{2}$ de $1708 \mu \mathrm{L} \mathrm{g}^{-1}$, após $6 \mathrm{~h}$ de irradiação, e $230 \mu \mathrm{L} \mathrm{g}^{-1}$ quando utilizou-se $\mathrm{TiO}_{2}$, nas mesmas condições acima.

A boa atividade do material se deveu à heterojunção de $\mathrm{CdS}$ em fase hexagonal com tamanho de partícula de $8 \mathrm{~nm}$ (bandgap de 2,3 $\mathrm{eV}$ ), que ejetou elétrons no $\mathrm{TiO}_{2}$ após a irradiação no visível, fazendo com que houvesse separação de cargas (elétron - lacuna) neste último semicondutor, conforme mostrado na Figura 6 abaixo:

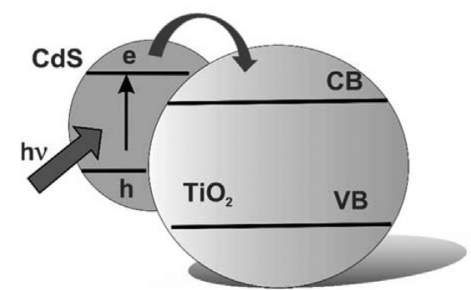

Figura 6. Esquema de separação de cargas utilizando-se a heterojunção de semicondutores Reimpresso da Ref. 60 com permissão. Copyright Willey-VCH Verlag $G m b H \& K G a A$ 


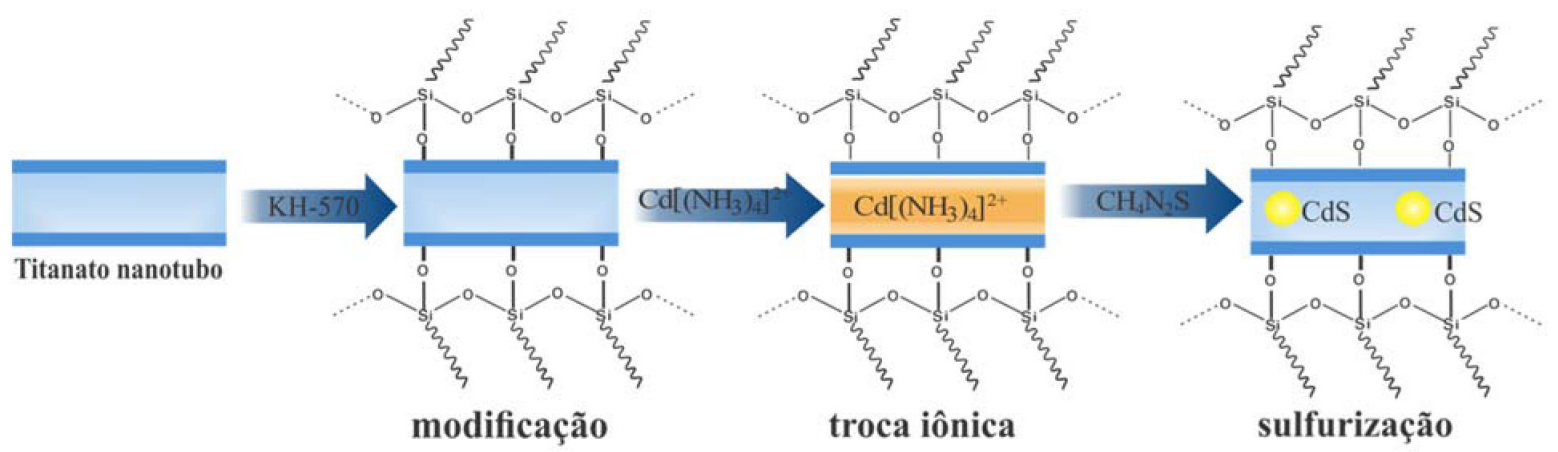

Figura 7. Esquema de deposição de nanopartículas de CdS em cavidades de TNT's. Reimpresso da Ref. 61 com permissão. Copyright IOP Publishing

Na mesma linha de pesquisa anterior, Long et al. ${ }^{61}$ realizaram a síntese de titanotubos (TNT), nos quais foram formadas nanopartículas confinadas de CdS a partir da sulfurização com tiouréia, conforme a Figura 7

Para efeito de comparação, as nanopartículas de CdS foram depositadas no exterior do TNT. Os materiais foram testados para geração fotacatalítica de $\mathrm{H}_{2}$ utilizando um reator de Pyrex irradiado com uma lâmpada de xenônio de $300 \mathrm{~W}$, utilizando filtros para remoção de radiação UV. O material foi disperso em água contendo $\mathrm{Na}_{2} \mathrm{~S}$ e $\mathrm{Na}_{2} \mathrm{SO}_{3}$ e foi adicionado $\mathrm{H}_{2} \mathrm{PtCl}_{6}$ para fotodeposição de platina.

$\mathrm{O}$ resultado de geração de $\mathrm{H}_{2}$ está mostrado na Figura 8 , na qual se pode observar a diferença de atividade entre o material com CdS na área externa do titanotubo (ex-TNTs) e CdS depositado dentro do TNT (in-TNTs)

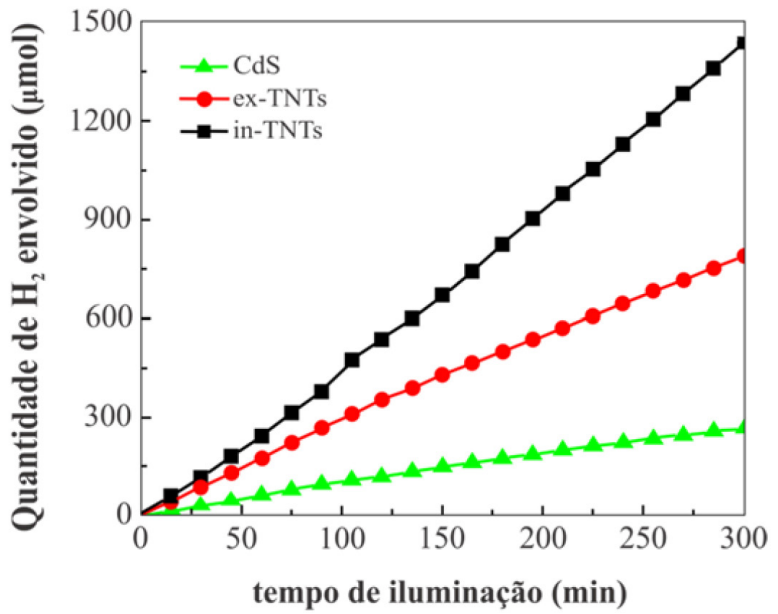

Figura 8. Evolução de hidrogênio utilizando titanotubos com CdS depositado. Reimpresso da Ref. 61 com permissão. Copyright IOP Publishing

A boa atividade fotocatalítica do in-TNTs foi atribuída pelos autores à baixa recombinação elétron/lacuna. ${ }^{61}$

Pérez-Larios et al. ${ }^{62}$ comprovaram que a mistura de óxidos semicondutores $\mathrm{TiO}_{2}-\mathrm{ZnO}$ aumenta a atividade fotocatalítica para geração de hidrogênio em soluções água:etanol (1:1) irradiadas com luz em $254 \mathrm{~nm}$. Os pesquisadores concluíram que a atividade destes óxidos em contato é seis vezes maior que a atividade do $\mathrm{TiO}_{2}$ sozinho, graças à transferência de elétrons do $\mathrm{ZnO}$ para o $\mathrm{TiO}_{2}$.

$\mathrm{Xu}$ e $\mathrm{Cao}^{63}$ sintetizaram, por método hidrotérmico, um compósito a base de $\mathrm{MoS}_{2} / \mathrm{CdS}$ e testaram o material para fotólise da água. Uma massa de $0,1 \mathrm{~g}$ do material em uma solução aquosa de ácido lático foi exposta a uma lâmpada de xenônio de $300 \mathrm{~W}$ equipada com filtro para luz UV. Os pesquisadores realizaram uma proposição para o mecanismo, cujo esquema está mostrado na Figura 9.

Sob excitação pela luz, elétrons e lacunas são geradas na banda de

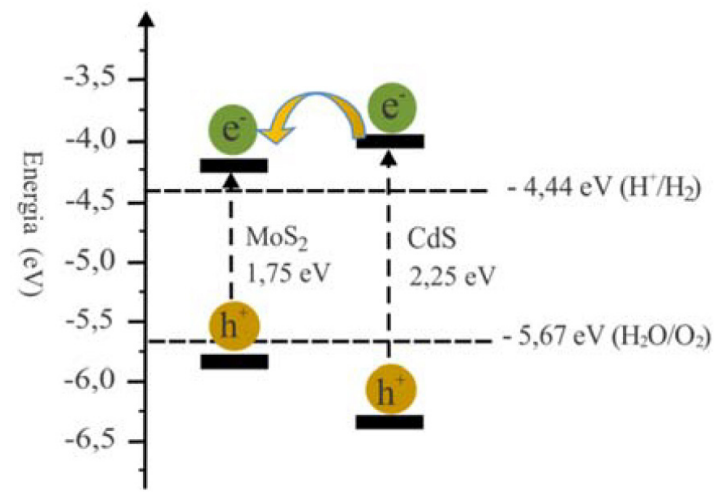

Figura 9. Proposta de mecanismo para geração fotocatalítica de hidrogênio utilizando o compósito CdS/MoS $\mathrm{S}_{2}$ como catalisador. Os valores de energia no gráfico são em relação ao vácuo. Adaptada da Ref. 63

condução e de valência do CdS, respectivamente. Devido à heterojunção, os elétrons fotogerados no CdS (microesferas com diâmetro de 1-6 $\mu \mathrm{m}$ ) são injetados no $\mathrm{MoS}_{2}$, realizando a separação elétron/lacuna e evitando a recombinação. A velocidade de produção de hidrogênio aumentou cerca de 17 vezes com a presença de $\mathrm{MoS}_{2}$ em relação ao CdS sozinho, com velocidade de 4,06 $\mathrm{mmol} \mathrm{g}^{-1} \mathrm{~h}^{-1}$.

Ainda utilizando heterojunção, Li et al. ${ }^{64}$ utilizaram $0,2 \mathrm{~g} \mathrm{CuInS} \mathrm{S}_{2} /$ $\mathrm{TiO}_{2}$ disperso numa mistura de $80 \mathrm{~mL}$ de água destilada e $20 \mathrm{~mL}$ de etanol e a suspensão foi irradiada com lâmpada de xenônio de 300 W, simulando a luz solar. Os autores obtiveram uma produção de hidrogênio ótima com o teor de 2,5\% em massa de $\mathrm{CuInS}_{2}$. Além disso, este material foi cerca de 7 vezes mais ativo que o $\mathrm{TiO}_{2}$ puro. De acordo com os autores, a heterojunção $\mathrm{CuInS}_{2} / \mathrm{TiO}_{2}$ promoveu a transferência de carga de forma fotoinduzida do semicondutor $\mathrm{CuInS}_{2}$ para o $\mathrm{TiO}_{2}$, devido ao primeiro ter um bandgap baixo o suficiente para excitação dos elétrons na região do visível. Esta transferência de elétrons para o $\mathrm{TiO}_{2}$ na presença do reagente de sacrifício causou a separação elétron/lacuna, evitando a recombinação no anatásio. Este processo também tornou a atividade fotocatalítica estável por um longo período de tempo.

De maneira similar à estratégia de heterojunção de semicondutores, pesquisas recentes estão se baseando na utilização de sensibilizadores, moléculas organometálicas ou simplesmente moléculas orgânicas. Abe et al., ${ }^{65}$ por exemplo, estudaram a sensibilização de $\mathrm{TiO}_{2}$ utilizando Eosina Y na presença de dietilamina para geração de $\mathrm{H}_{2}$. Por outro lado, Zheng et al. ${ }^{66}$ sintetizaram um composto de coordenação, o [RuL(bpy $\left.)_{2}\right]\left(\mathrm{PF}_{6}\right)_{2}$, sendo $\mathrm{L}=$ ácido 2-hidroxi-5-(imidazo[4,5-f]-1,10-fenantrolina) benzóico e bpy =2,2'-bipiridina, chamado pelos autores de TM1. O composto organometálico foi depositado na superfície do $\mathrm{TiO}_{2}$, utilizando Pt como co-catalisador. $\mathrm{O}$ teste fotocatalítico foi realizado utilizando-se materiais com diferentes concentrações de TM1, suspensos em água com trietanolamina 


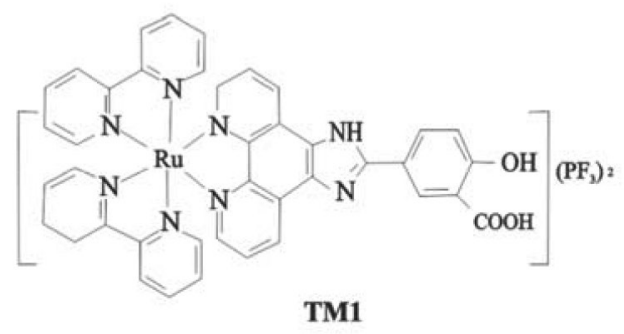

(a)

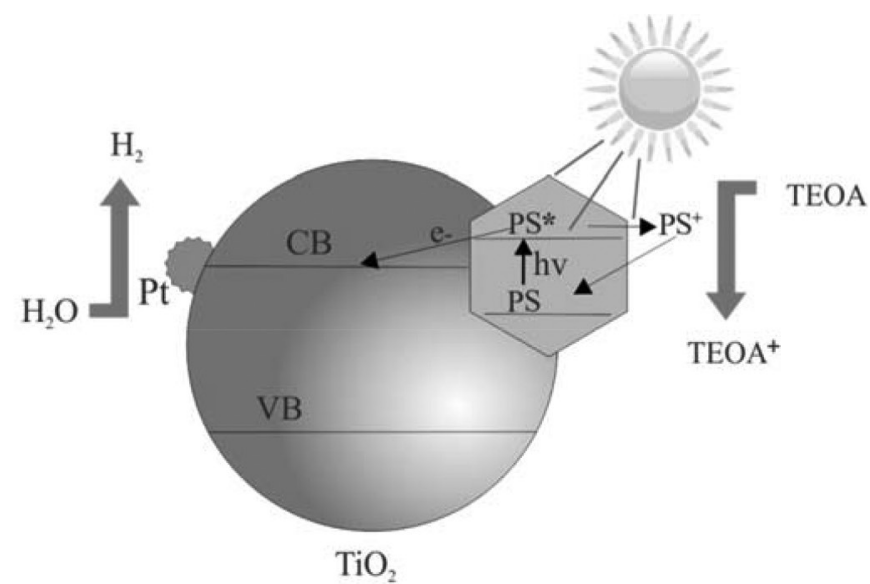

(b)

Figura 10. Mecanismo de geração de $\mathrm{H}_{2}$ por sensibilização do $\mathrm{TiO}_{2}$ (b) utilizando TM1(a). Adaptada da Ref. 66

(TEOA), e irradiados com lâmpada de Xe de 300 W equipada com filtro para $\lambda<420 \mathrm{~nm}$.

$\mathrm{O}$ mecanismo de sensibilização proposto para o $\mathrm{TiO}_{2}$ está baseado na conjugação $\mathrm{TiO}_{2}$-sensibilizador e geradora de efeito antena (similar à fotossíntese de plantas). Como mostrado na Figura 10, PS (do inglês photosystem) que representa a molécula organometálica, é excitado por luz visível a PS*, que injeta elétrons na banda de condução do $\mathrm{TiO}_{2}$, fazendo a separação de cargas no semicondutor, com consequente transferência de elétrons para a Pt e início do processo catalítico. Além disso, a presença da trietanolamina evitou a recombinação elétron/lacuna. ${ }^{66}$

Similarmente, Rao et al ${ }^{67}$ utilizaram dois complexos de níquel para a fotólise da água, chamados de $\left[\mathrm{Ni}\left(\mathrm{Hqt}_{2}(4,4\right.\right.$ '-Z-2, 2'-bpy)] (Hqt $=8$-quinolinetiol, sendo $\mathrm{Z}=-\mathrm{H}$ [1] ou $-\mathrm{CH}_{3}$ [2], bpy = bipiridina. $\mathrm{O}$ teste fotocatalítico foi realizado com os catalisadores dissolvidos na solução, ou seja, do tipo homogêneo. O catalisador foi adicionado a uma mistura de $50 \%$ de etanol em água e irradiado com lâmpada de xenônio de $300 \mathrm{~W}$ ( $\lambda>420 \mathrm{~nm}$ ). Os resultados do trabalho mostraram boa atividade dos materiais e concluiu-se que o hidrogênio gerado resultou da formação de hidretos intermediários.

$\mathrm{Na}$ tentativa de viabilizar a utilização do $\mathrm{TiO}_{2}$ na fotocatálise, diversas alternativas de modificações como as citadas acima estão sendo estudadas. Essas modificações estão relacionadas com as propriedades elétricas e estruturais do material. $\mathrm{O}$ aumento da eficiência do semicondutor no fenômeno de Water Splitting é dependente da estrutura cristalina, da morfologia, da estrutura de bandas e da faixa de absorção da radiação eletromagnética. ${ }^{15}$ Os óxidos de metais de transição, quando incorporados nas estruturas de zeólitas e de peneiras moleculares mesoporosas, geram atividade não somente para reações catalíticas, mas também para as reações fotocatalíticas. ${ }^{68}$

Em particular, óxidos de metais de transição altamente dispersos, tais como os de titânio, vanádio, e molibdênio têm exibido elevada reatividade fotocatalítica para várias reações, devido a processos de transferência de carga, principalmente quando expostos à irradiação UV, de acordo com o Esquema 1:

$$
\left[M^{n+}-O^{2-}\right] \stackrel{h v}{\longrightarrow}\left[M^{(n-1)+}-O^{-}\right]^{*} \quad(\mathrm{M}: \mathrm{Ti}, \mathrm{V}, \mathrm{Cr}, \mathrm{Mo} \ldots)
$$

Esquema 1. Formação de um estado excitado pela transferência de carga em óxidos de metais de transição, sob irradiação de $l u z^{69}$

Cui et al. ${ }^{70}$ estudaram a geração fotocatalítica de $\mathrm{H}_{2}$ por fotocatálise de vapor de metanol empregando zeólita beta com titânio incorporado em sua estrutura (Ti-beta). A reação fotocatalítica foi processada num reator de quartzo com $6 \mathrm{~mL}$ de capacidade e utilizando uma lâmpada de mercúrio de baixa pressão ( $4 \mathrm{~W}$ e comprimento de onda principal em $254 \mathrm{~nm}$ ). A quantidade de hidrogênio gerada foi de $0,01204 \mathrm{mmol} \mathrm{g}^{-1}$ após $3 \mathrm{~h}$ de iluminação para a zeólita Ti-beta e foi aproximadamente 10 vezes maior que em uma zeólita impregnada com $\mathrm{TiO}_{2}$. A maior atividade encontrada para a zeólita Ti-beta foi relacionada à alta adsorção de metanol na superfície do material e também em função da incorporação de Ti na estrutura da zeólita. Os autores propuseram um mecanismo de reação, no qual se sugere a formação do estado excitado $\left(\mathrm{Ti}^{3+}-\mathrm{O}^{-}\right)^{*}$.

$\mathrm{Liu}^{71}$ estudou a geração de $\mathrm{H}_{2}$ pela decomposição da água utilizando MCM-41 modificada com zircônio (Zr-MCM-41), em suspensão de água pura e irradiada com uma lâmpada de mercúrio de alta pressão, com potência de $400 \mathrm{~W}$. A decomposição da molécula de $\mathrm{H}_{2} \mathrm{O}$ produziu $112 \mathrm{mmol}$ de $\mathrm{H}_{2} \mathrm{em} 5 \mathrm{~h}$ de reação. O trabalho mostrou que a atividade fotocatalítica do Zr-MCM-41 em luz UV é cerca de 2,5 vezes maior que a do $\mathrm{ZrO}_{2}$, devido à alta dispersão da zircônia na superfície da peneira molecular.

Shen e $\mathrm{Guo}^{72}$ verificaram a formação de $\mathrm{H}_{2}$ usando peneiras moleculares MCM-41 modificadas com Ti e Cr, sintetizadas pelo método hidrotérmico. A atividade fotocatalítica foi avaliada utilizando 0,2 g do material disperso em $200 \mathrm{~mL}$ de solução aquosa com ácido fórmico (proporção de 3:1), irradiada com uma lâmpada de xenônio de $350 \mathrm{~W}$ equipada com filtro para $\lambda<430 \mathrm{~nm}$. A peneira molecular com razão molar $\mathrm{Si} / \mathrm{Cr}=100$ apresentou maior atividade fotocatalítica $\left(146,4 \mu \mathrm{mol} \mathrm{g}^{-1} \mathrm{~h}^{-1}\right)$. A alta atividade na região do visível foi associada à baixa recombinação elétron/lacuna em função da presença de mais de um centro metálico.

Utilizando uma peneira da mesma família da MCM-41, Zhao et al. ${ }^{73}$ realizaram a síntese hidrotérmica da MCM-48 com Ti incorporado nas razões molares $\mathrm{Si} / \mathrm{Ti}=25-200$ e testaram a atividade fotocatalítica para geração de hidrogênio de uma suspensão de $2 \mathrm{mg}$ do material em 1,6 mL de água e 0,4 mL de metanol, utilizando uma lâmpada de xenônio de $500 \mathrm{~W}$ equipada com filtro para eliminar os comprimentos de onda maiores que $230 \mathrm{~nm}$ e menores que $380 \mathrm{~nm}$. A atividade fotocatalítica foi de $12,75 \mathrm{mmol} \mathrm{h}^{-1}\left(\mathrm{~g}_{\mathrm{Ti}}\right)^{-1}$ para a peneira molecular Ti-MCM-48-1-200. Neste caso, espécies de Ti tetraedricamente coordenadas foram associadas aos sítios ativos para a reação fotocatalítica na região do UV, na ausência de Pt como co-catalisador.

Em outro estudo, Liu et al. ${ }^{74}$ sintetizaram peneiras moleculares CdS/M-MCM-41 (M = Zr, Ti) pelo método de troca iônica e testaram para a produção fotocatalítica de $\mathrm{H}_{2}$. Diferentes soluções aquosas de cinco reagentes de sacrifício (etanol, ácido acético, ácido propanóico, ácido butírico e trietanoamina) com os fotocalisadores foram expostas 
à radiação visível, utilizando uma lâmpada de xenônio de 300 W. Os resultados de difratometria de raios-X de baixo ângulo dos materiais sintetizados mostraram que o Ti influencia mais na desorganização da estrutura mesoporosa da MCM-41 do que o Zr. As maiores atividades fotocatalíticas encontradas foram para $\mathrm{CdS} / \mathrm{Zr}(0,005)-\mathrm{MCM}-41$ e CdS/Ti(0,02)-MCM-41 (onde o valor entre parênteses representa a razão $\mathrm{M} / \mathrm{Si}$ ) com velocidades de geração de $\mathrm{H}_{2}$ de $6,058 \mu \mathrm{mol} \mathrm{h}^{-1} \mathrm{e}$ $9,422 \mu \mathrm{mol} \mathrm{h} \mathrm{h}^{-1}$, respectivamente. Ainda, os pesquisadores verificaram melhor atividade para geração de hidrogênio quando se utilizou trietanolamina como reagente de sacrifício.
Outros resultados de trabalhos recentes são compilados na Tabela 1, na qual são mostrados os materiais, condições do experimento e a atividade fotocatalítica em função da geração de hidrogênio.

\section{CONSIDERAÇÕES FINAIS}

A crise energética e o agravamento do efeito estufa impulsionaram pesquisas para a produção de hidrogênio. Há na literatura e também em fase de comercialização diversas metodologias para sua geração, dentre elas a redução da água usando fotocatalisadores. Apesar de

Tabela 1. Trabalhos envolvendo a geração de $\mathrm{H}_{2}$ por fotocatálise

\begin{tabular}{|c|c|c|c|c|c|}
\hline Material & Irradiação & Reagente Sacrifício & $\begin{array}{l}\text { Outras Condições Experi- } \\
\text { mentais }\end{array}$ & Atividade & Referência \\
\hline $\mathrm{InP} / \mathrm{Pt}$ & $\begin{array}{l}\mathrm{Hg} \text { alta pressão, } 250 \mathrm{~W}, \\
\qquad>400 \mathrm{~nm}\end{array}$ & $\mathrm{SO}_{3}^{2-} 0,5 \mathrm{~mol} \mathrm{~L}^{-1}$ & $30 \mathrm{mg}$ & $2-5 \mu \mathrm{mol} \mathrm{h}^{-1}$ & 75 \\
\hline \multicolumn{6}{|l|}{$\mathrm{In}_{0,90} \mathrm{Ni}_{0,10} \mathrm{TaO}_{4}$} \\
\hline$/ \mathrm{NiO}_{\mathrm{x}}$ & $\mathrm{Xe}, 300 \mathrm{~W}$ & - & $0,5 \mathrm{~g} / 250 \mathrm{~mL} \mathrm{H}_{2} \mathrm{O}$ & $16,6 \mu \mathrm{mol} \mathrm{h}^{-1}$ & 76 \\
\hline $\mathrm{TiO}_{2} / 1,2 \% \mathrm{Cu}$ & $\begin{array}{l}16 \text { Lâmpadas UV, } 12 \mathrm{~W}, \\
\lambda_{\max }=300 \mathrm{~nm}\end{array}$ & $\mathrm{CH}_{3} \mathrm{OH}: \mathrm{H}_{2} \mathrm{O}(1,4: 1)$ & 1,25 g em $1 \mathrm{~L}$ solução & $\sim 6300 \mu \mathrm{mol}(30 \mathrm{~min})^{-1}$ & 77 \\
\hline $\mathrm{InNiCr}_{2} \mathrm{Ti}_{10} \mathrm{O}_{42} / \mathrm{Pt}$ & $\mathrm{Xe}, 200 \mathrm{~W}, \lambda \geq 420 \mathrm{~nm}$ & $\begin{array}{l}50 \mathrm{~mL} \mathrm{CH}_{3} \mathrm{OH}+ \\
220 \mathrm{~mL} \mathrm{H}_{2} \mathrm{O}\end{array}$ & 0,5g em $270 \mathrm{~mL}$ solução & $\sim 295 \mu \mathrm{mol}(44 \mathrm{~h})^{-1}$ & 78 \\
\hline $\mathrm{CdS} / \mathrm{TiO}_{2}$ & $\mathrm{Hg}, 500 \mathrm{~W}, \lambda \geq 420 \mathrm{~nm}$ & $\mathrm{Na}_{2} \mathrm{~S}+\mathrm{Na}_{2} \mathrm{SO}_{3}$ & 0,1 g em 100 mL solução & $422,4 \mu \mathrm{mol} \mathrm{h}^{-1}$ & 79 \\
\hline $\mathrm{CdS} / \mathrm{TiO}_{2}$ (nanotubo) & $\mathrm{Xe}, 350 \mathrm{~W}, \lambda>400 \mathrm{~nm}$ & $\mathrm{Na}_{2} \mathrm{~S}+\mathrm{Na}_{2} \mathrm{SO}_{3}$ & 0,1 g em 50 mL solução & $1708 \mu \mathrm{L} \mathrm{g} \mathrm{g}^{-1}(6 \mathrm{~h})^{-1}$ & 59 \\
\hline $\mathrm{Au} / \mathrm{KTiNbO}_{5}$ & $\begin{array}{l}\text { Halógena, } 400 \mathrm{~W}, \\
\lambda_{\text {máx }}=360 \mathrm{~nm}\end{array}$ & Metanol: $\mathrm{H}_{2} \mathrm{O}(1: 5)$ & 0,2 g em 100 mL solução & $3522 \mu \mathrm{mol} \mathrm{g} \mathrm{g}^{-1} \mathrm{~h}^{-1}$ & 80 \\
\hline $\mathrm{Ni} @ \mathrm{C} / \mathrm{TiO}_{2}$ & $\mathrm{Xe}, 300 \mathrm{~W}, \lambda \geq 420 \mathrm{~nm}$ & \multicolumn{2}{|c|}{ Trietanolamina $15 \%$ (v/v) 0,15g em 100 mL solução } & $300 \mu \mathrm{mol} \mathrm{h}{ }^{-1}$ & 81 \\
\hline $\mathrm{Pt} / \mathrm{ZnIn}_{2} \mathrm{~S}_{4}$ & $\mathrm{Hg}, 400 \mathrm{~W} \lambda \geq 420 \mathrm{~nm}$ & Trietanolamina & $\begin{array}{c}0,05 \text { g em } 100 \mathrm{~mL} \\
\text { solução }\end{array}$ & $160 \mu \mathrm{mol}(10 \mathrm{~h})^{-1}$ & 82 \\
\hline $\mathrm{Pt} / \mathrm{Cd}_{1-\mathrm{x}} \mathrm{Zn}_{\mathrm{x}} \mathrm{S} / \mathrm{ZnO} / \mathrm{Zn}(\mathrm{OH})_{2}$ & $\mathrm{Hg}, 1000 \mathrm{~W}, \lambda \geq 420 \mathrm{~nm}$ & Glicerol & $\begin{array}{l}0,77 \text { g em } 1000 \mathrm{~mL} \\
\text { solução }\end{array}$ & $449 \mu \mathrm{mol} \mathrm{g}^{-1} \mathrm{~h}^{-1}$ & 83 \\
\hline $\mathrm{RuO}_{2} / \mathrm{TiSi}_{2} /$ grafeno & $\mathrm{Xe}, 150 \mathrm{~W}, \lambda \geq 420 \mathrm{~nm}$ & - & $0,1 \mathrm{~g} / 50 \mathrm{~mL} \mathrm{H}_{2} \mathrm{O}$ & $97,5 \mu \mathrm{mol} \mathrm{g} \mathrm{g}^{-1} \mathrm{~h}^{-1}$ & 84 \\
\hline $\mathrm{PdS} / \mathrm{CdS}$ & $\mathrm{Xe}, 300 \mathrm{~W}, \lambda>430 \mathrm{~nm}$ & $\mathrm{Na}_{2} \mathrm{~S}+\mathrm{Na}_{2} \mathrm{SO}_{3}$ & 0,2 g em 200 mL solução & $890,3 \mu \mathrm{mol} \mathrm{h}{ }^{-1}$ & 85 \\
\hline $\mathrm{ZnS}-\mathrm{CuS}-\mathrm{CdS}$ & $\mathrm{Xe}, 150 \mathrm{~W}, \lambda \geq 420 \mathrm{~nm}$ & $\mathrm{Na}_{2} \mathrm{~S}$ & 0,1 g em $150 \mathrm{~mL}$ solução & $838 \mu \mathrm{mol} \mathrm{g}{ }^{-1} \mathrm{~h}^{-1}$ & 86 \\
\hline $\mathrm{Pt} / \mathrm{Ni}_{2} \mathrm{O}_{3}-\mathrm{SnO}_{2}$ & $\mathrm{~W}, 300 \mathrm{~W}$ & Etanol 20\% (v/v) & 0,2 g em 100 mL solução & $0,9 \mu \mathrm{mol} \mathrm{g}{ }^{-1} \mathrm{~h}^{-1}$ & 87 \\
\hline $\mathrm{AuPd} / \mathrm{TiO}_{2}$ nanofibra & $\begin{array}{l}\text { Simulador solar (67005, } \\
\text { Newport Corp.) }\end{array}$ & $\begin{array}{l}\text { Ácido fórmico } \\
2,7 \mathrm{~mol} \mathrm{~L}^{-1}\end{array}$ & 5 mg em 10 mL solução & $88,5 \mu \mathrm{mol} \mathrm{h}^{-1}$ & 88 \\
\hline $\mathrm{CdS} / \mathrm{WO}_{3}$ & $\begin{array}{l}4 \text { lamp. Fluorescentes } \\
15 \mathrm{~W}\end{array}$ & $\begin{array}{l}\text { Ácido fórmico } \\
10^{-3} \mathrm{~mol} \mathrm{~L}^{-1}\end{array}$ & $0,1 \mathrm{~g} \mathrm{em} 50 \mathrm{~mL}$ solução & $4,74 \mu \mathrm{mol}(6 \mathrm{~h})^{-1}$ & 89 \\
\hline $\mathrm{Mo}_{2} \mathrm{~S}$-piridina- $\mathrm{gC}_{3} \mathrm{~N}_{4}$ & $\mathrm{Xe}, 300 \mathrm{~W}, \lambda>420 \mathrm{~nm}$ & Trietanolamina $10 \%(\mathrm{v} / \mathrm{v})$ & $\begin{array}{c}50 \text { mg em } 100 \mathrm{~mL} \\
\text { solução }\end{array}$ & $25 \mu \mathrm{mol} \mathrm{h}^{-1}$ & 90 \\
\hline $\mathrm{Pt} / \mathrm{TiO}_{2}$ (esferas) & $\mathrm{Hg}, 400 \mathrm{~W} \lambda \geq 420 \mathrm{~nm}$ & Metanol 10\% (v/v) & 0,5 g em $250 \mathrm{~mL}$ solução & $1023,71 \mu \mathrm{mol} \mathrm{g} \mathrm{g}^{-1} \mathrm{~h}^{-1}$ & 91 \\
\hline $\mathrm{S} / \mathrm{TiO}_{2}$ & $\begin{array}{l}\text { Simulador solar } 100 \mathrm{~mW} / \\
\mathrm{cm}^{2} \text { (Oriel) }\end{array}$ & Metanol 20\% (v/v) & $\begin{array}{c}100 \text { mg em } 100 \text { mL } \\
\text { solução }\end{array}$ & $163,9 \mu \mathrm{mol} \mathrm{g}{ }^{-1} \mathrm{~h}^{-1}$ & 92 \\
\hline Filme $\mathrm{CuO}_{x} / \mathrm{TiO}_{2}$ & Xe, $300 \mathrm{~W}$ & Metanol 10\% (v/v) & $100 \mathrm{~mL}$ & $53 \mathrm{mmol} \mathrm{m}^{-2} \mathrm{~h}^{-1}$ & 93 \\
\hline $\mathrm{Au} / \mathrm{TiO}_{2}$ (nanotubo) & $\mathrm{Hg}-\mathrm{Xe} 150 \mathrm{~W}$ & Metanol 12,5\% (v/v) & $\begin{array}{l}\text { Área fotocatalisador - } \\
\qquad 1,23 \mathrm{~cm}^{2}\end{array}$ & $1,78 \mu \mathrm{mol} \mathrm{cm} \mathrm{cm}^{-2} \mathrm{~h}^{-1}$ & 94 \\
\hline $\mathrm{Cu} / \mathrm{ZnS}$ & $\mathrm{Xe}, 350 \mathrm{~W}, \lambda \geq 400 \mathrm{~nm}$ & $\mathrm{Na}_{2} \mathrm{~S}\left(0,05-0,1 \mathrm{~mol} \mathrm{~L}^{-1}\right)$ & $\begin{array}{c}100 \mathrm{~mL} \text { solução } 0,3-0,5 \\
\mathrm{~g} \mathrm{~L}^{-1}\end{array}$ & $973,1 \mu \mathrm{mol} \mathrm{g}{ }^{-1} \mathrm{~h}^{-1}$ & 95 \\
\hline $\begin{array}{l}\beta \text {-BiTaO } \\
\text { zadores }\end{array}$ & $\mathrm{Xe}, 500 \mathrm{~W}, \lambda>418 \mathrm{~nm}$ & Isopropanol 30\% (v/v) & 60 mg em 60 mL solução & $\sim 12 \mu \mathrm{mol} \mathrm{g}{ }^{-1}(7 \mathrm{~h})^{-1}$ & 96 \\
\hline $\mathrm{TiO}_{2}$ - RGO (óxido de grafeno) & Xe-Hg, 300 W & Etanol 25\% (v/v) & 4 mg em 8 mL solução & $3 \mathrm{mmol} \mathrm{g}^{-1}(2,5 \mathrm{~h})^{-1}$ & 97 \\
\hline $\mathrm{Au} / \mathrm{ZnO}$ & $\mathrm{Xe}-\mathrm{Hg}, 300 \mathrm{~W}$ & Etanol 25\% (v/v) & 7 mg em 8 mL solução & $427 \mu \mathrm{mol} \mathrm{g} \mathrm{g}^{-1} \mathrm{~h}^{-1}$ & 98 \\
\hline $\mathrm{CdS} / \mathrm{TiO}_{2}$ (nanotubo) & $\begin{array}{l}\text { Fonte não informada, } \\
\qquad \lambda>420\end{array}$ & $\mathrm{~S}^{-2}+\mathrm{SO}_{3}^{-2} 0,1 \mathrm{~mol} \mathrm{~L}^{-1}$ & - & $0,3 \mu \mathrm{mol} \mathrm{cm} \mathrm{cm}^{-2} \mathrm{~h}^{-1}$ & 99 \\
\hline $\mathrm{Au} / \mathrm{TiO}_{2}$ (nanotubo) & $\mathrm{Xe}-\mathrm{Hg}, 300 \mathrm{~W}$ & Formaldeído $1 \%(\mathrm{~m} / \mathrm{m})$ & - & $\begin{array}{l}0,0033 \mu \mathrm{mol} \mathrm{h}^{-1} \\
(\lambda \geq 400 \mathrm{~nm}) \\
0,0029 \mu \mathrm{mol} \mathrm{h}-1 \\
(\lambda \geq 450 \mathrm{~nm})\end{array}$ & 100 \\
\hline $\mathrm{Ag} / \mathrm{TiO}_{2}$ & $\mathrm{Hg}, 250 \mathrm{~W}$ & Metanol 20\% (v/v) & 0,2 g em 200 mL solução & $180 \mu \mathrm{mol}(4 \mathrm{~h})^{-1}$ & 101 \\
\hline $\mathrm{TiO}_{2}$ Mesoporoso & $\mathrm{Hg}-\mathrm{Xe}, 240 \mathrm{~W}$ & Etanol 33\% (v/v) & 8 mg em 6 mL solução & $1304 \mu \mathrm{mol}(2,5 \mathrm{~h})^{-1}$ & 102 \\
\hline $\mathrm{Co}(\mathrm{OH})_{2} / \alpha-\mathrm{Fe}_{2} \mathrm{O}_{3}$ & $\mathrm{Hg}-\mathrm{Xe}, 240 \mathrm{~W}$ & Etanol 23,8\% (v/v) & $4 \mathrm{mg}$ em 8 mL solução & $546 \mu \mathrm{mol} \mathrm{g}{ }^{-1} \mathrm{~h}^{-1}$ & 103 \\
\hline
\end{tabular}


ser um processo eficiente, o uso de luz UV artificial ainda torna o processo fotocatalítico pouco aplicável para escalas industriais. $\mathrm{Na}$ intenção de prover condições para a mudança neste paradigma, pesquisas recentes estão voltadas ao desenvolvimento de materiais com aproveitamento cada vez maior da luz visível do espectro solar, com o objetivo claro de se utilizar uma fonte de energia limpa, segura, renovável e principalmente abundante. Além da dificuldade em utilizar luz UV, aqueles poucos materiais que absorvem luz visível apresentam um potencial de redução da banda de condução mais positivo que o potencial de redução do $\mathrm{H}^{+}$a $\mathrm{H}_{2}$ e ainda requerem o uso de reagentes de sacrifício como doares irreversíveis de elétrons para evitar a recombinação elétron/lacuna.

Pesquisas recentes apontam soluções e alternativas para mitigar os problemas citados acima. Por exemplo, no Esquema $\mathrm{Z}$ dispensa-se a utilização de reagentes de sacrifício. Neste caso, os mediadores atuam de forma reversível. De todo modo, existe uma forte tendência na utilização de poluentes como reagentes de sacrifício, já que deste modo é possivel reduzir a quantidade de resíduos paralelamente à geração de $\mathrm{H}_{2}$. A resolução da diminuição do bandgap e a busca de potenciais adequados para a fotorredução da água são sugeridos, através de abordagens como: a dopagem de óxidos com íons de metais de transição com configuração eletrônica do tipo $\mathrm{d}^{\mathrm{n}}(0<\mathrm{n}<10)$; controle da banda de valência de óxidos metálicos usando orbitais $\mathrm{p}$ de um ânion ou os orbitais s de íons de metal do bloco p e sensibilização espectral.

\section{AGRADECIMENTOS}

Os autores agradecem à FAPERJ (proc. E-26/010.002631/2014) e à FAPES (proc. 67677258).

\section{REFERÊNCIAS}

1. International Energy Agency; Prospects For Hydrogen And Fuel Cells, Paris, França, 2005. https://www.iea.org/publications/freepublications/ publication/hydrogen.pdf, acessada em Dezembro 2016.

2. International Energy Agency; World Energy Outlook, Paris, França, 2008. http://www.worldenergyoutlook.org/media/weowebsite/2008-1994/weo2008.pdf, acessada em Dezembro 2016.

3. Greenpeace; [r]evolution a sustainable world energy outlook, 2015. http://www.greenpeace.org/international/Global/international/ publications/climate/2015/Energy-Revolution-2015-Full.pdf, acessada em Dezembro 2016.

4. Smith, B.; Shantha, M. S.; Int. J. Chem. React. Eng. 2007, $5,1$.

5. Ni, M.; Leung, M. K. H.; Leung, D. Y. C.; Renewable Sustainable Energy Rev. 2007, 11, 401.

6. Patsoura, A.; Kondarides, D. I.; Verykios, X. E.; Catal. Today. 2007, 124, 94.

7. Strataki, N.; Bekiari, V.; Kondarides, D. I.; Lianos, P.; Appl. Catal., B. 2007, 77, 184.

8. Fujishima, A.; Honda, K.; Nature 1972, 238, 37.

9. Ellis, A. B.; Geselbracht, M. J.; Johnson, B. J.; Linsensky, G. C.; Robinson, W. R.; General Chemistry, A Materials Science Companion, American Chemical Society: Washington DC, 1993.

10. Hoffmann, R.; Solids and Surfaces, Wiley-VCH, New York, 1988.

11. Smart, L.; Moore, Y. E. A.; Química Del Estado Sólido: Una Introducción, Wesley, Iberoamericana: Wilmington, 1995.

12. Cox, P. A.; The Electronic Structure and Chemistry of Solids, Oxford Univ. Press: Oxford, 1987.

13. Hoffmann, M. R.; Martin, S. T.; Choi, W. Y.; Bahnemann, D. W.; Chem. Rev. 1995, 95, 69.

14. Linsebigler, A. L.; Lu, G.; Yates, J. T. J.; Chem. Rev. 1995, 95, 735.

15. Leung, D. Y. C.; ChemSusChem 2010, 3, 681.
16. Sato, S.; White, J. M.; Chem. Phys. Lett. 1980, 72, 83.

17. Melo, M. O.; Silva, L. A.; J. Braz. Chem. Soc. 2011, 22, 1399.

18. Gonzalez-Elipe, A.; Munuera, G.; Soria, J.; J. Chem. Soc. 1979, 103, 4685 .

19. Anpo, M.; Takeuchi, M.; J. Catal. 2003, 216, 505.

20. Chen, X.; Shen, S.; Guo, L.; Mao, S. S.; Chem. Rev. 2010, 110, 6503.

21. Maeda, K.; Domen, K.; J. Phys. Chem. C 2007, 111, 7851.

22. Galinska, A.; Walendziewski, J.; Energy Fuels 2005, 19, 1143.

23. Hara, K.; Sayama, K.; Arakawa, H.; Appl. Catal., A 1999, 189, 127.

24. Kondarides, D. I.; Daskalaki, V. M.; Patsoura, A.; Verykios, X. E.; Catal. Lett. 2008, 26, 122.

25. Bamwenda, G. R; Tsubota, S.; Nakamura, T.; Haruta, M.; J Photochem Photobiol A: Chem. 1995, 89, 177.

26. Lee, S. G.; Lee, S.; Lee, H. I.; Appl Catal, A 2001, 207, 173.

27. Li, Y. X.; Lu, G. X.; Li, S. B.; Chemosphere 2003, 52, 843.

28. Nada, A. A.; Barakat, M. H.; Hamed, H. A.; Mohamed, N. R.; Veziroglu, T. N.; Int. J. Hydrogen Energy. 2005, 30, 87.

29. Jang, J. S.; Kim, H. G.; Borse, P. H.; Lee, J. S.; Int. J. Hydrogen Energy. 2007, 32, 4786.

30. Speltini, A.; Sturini, M.; Maraschi, F.; Dondi, D.; Fisogni, G.; Annovazzi, E.; Profumo, A.; Buttafava, A.; Int. J. Hydrogen Energy. 2015, 40, 4303 .

31. Speltini, A.; Sturini, M.; Maraschi, F.; Dondi, D.; Serra, A.; Profumo, A.; Buttafava, A.; Albini, A.; Int. J. Hydrogen Energy. 2014, 39, 11433.

32. Ravishankar, T. N.; Ramakrishnappa, T.; Nagabhushana, H.; Souza, V. S.; Dupont, J.; Nagaraju, G. New J. Chem. 2015, 39, 1421.

33. Souza, E. A.; Silva, L. A.; J. Environ. Chem. Eng. 2016, 4, 2114.

34. Daskalaki, V. M.; Kondarides, D. I.; Catal. Today. 2009, 144, 75.

35. Liu, R.; Yoshida, H.; Fujita, S.; Arai, M.; Appl. Catal., B 2014, 144, 41.

36. Languer, M. P.; Scheffer, F. R.; Feil, A. F.; Baptista, D. L.; Migowski, P.; Machado, G. J.; Moraes, D. P.; Dupont, J.; Teixeira, S. R.; Weibel, D. E. Int. J. Hydrogen Energy. 2013, 38, 14440.

37. Fujita, S.; Kawamori, H.; Honda, D.; Yoshida, H.; Arai, M.; Appl. Catal., B 2016, $181,818$.

38. Bard, A. J.; J. Photochem. 1979, 10, 59.

39. Maeda, K.; J. Photochem. Photobiol., C 2011, 12, 237.

40. Lo, C-C.; Huang, C-W.; Liao, C-H.; Wu, J.; Int. J. Hydrogen Energy. 2010, 35,1523 .

41. Sayama, K; Mukasa, K.; Abe, R.; Abe, Y.; Arakawa, H. A.; J. J. Photochem. Photobiol., A 2002, 148, 71.

42. Higashi, M.; Abe, R.; Takata, T.; Domen, K.; Chem. Mater. 2009, 21, 1543.

43. Yan, J.; Wu, H.; Chen, H.; Zhang, Y.; Zhang, F.; Liu, S. F.; Appl. Catal., B 2016, 191,130.

44. Scaife, D. E.; Solar Energy 1980, 25, 41.

45. Matsumura, M.; Saho, Y. H. Tsubomura.; J. Phys. Chem. 1983, 87, 3807.

46. Reber, J. F.; Meier, M.; J. Phys. Chem. 1986, 90, 824.

47. Erbs, W.; Desilvestro, J.; Borgarello, E.; Grätzel, M.; J. Phys. Chem. 1984, $88,4001$.

48. Williams, R.; J. Chem. Phys. 1960, 32, 1505.

49. Ellis, A. B.; Kaiser, S. W.; Bolts, J. M.; Wrighton, M. S.; J. Am. Chem. Soc. 1977, 99, 2839.

50. Kozlova, E. A.; Markovskaya, D. V.; Cherepanova, S. V.; Saraev, A. A.; Gerasimov, E. Y.; Perevalov, T. V.; Kaichev, V. V.; Parmon, V. N.; Int. J. Hydrogen Energy. 2014, 39,18758.

51. Bastos, S. A. L.; Lopes, P. A. L.; Santos, F. N.; Silva, L. A.; Int. J. Hydrogen Energy. 2014, 39, 14588.

52. Sato, J.; Saito, N.; Nishiyama, H.; Inoue, Y.; J. Phys. Chem. B 2001, 105, 2061.

53. Ye, J.; Zou, Z.; Oshikiri, M.; Matsushita, A.; Shimoda, M.; Imai, M. Shishido, T.; Chem. Phys. Lett. 2002, 356, 221.

54. Iwase, A.; Kato, H.; Kudo, A.; Catal. Lett. 2006, 108, 7. 
55. Zou, Z.; Ye, J.; Arakawa, H.; Int. J. Hydrogen Energy 2003, 28, 663.

56. Dholam, R.; Patel, N.; Adami, M.; Miotello, A.; Int. J. Hydrogen Energy 2009, 34, 5337.

57. Xiang, Q.; Yu, J.; Jaroniec, M. J. Phys. Chem. . 2011, 115, 7355.

58. Lan, Z.; Zhang, G.; Wang, X.; Appl. Catal., B 2016, 192, 116.

59. Yaojun, Z.; Yanpei, W.; Zhenhua, W.; Yaru, H.; Rare Met. Mater. Eng. 2009, 38, 1514.

60. Baker, D. R.; Kamat, P. V.; Adv. Funct. Mater. 2009, 19, 805.

61. Long, L.; Yu, X.; Wu, L.; Li, J.; Li, X.; Nanotechnology 2014, 25, 1.

62. Pérez-Larios, A.; Lopez, R.; Hernández-Gordillo, A.; Tzompantzi, F.; Gómez, R.; Torres-Guerra, L. M.; Fuel 2012, 100, 139.

63. Xu, J.; Cao, X.; Chem. Eng. J. 2015, 260, 642.

64. Li, C.; Xi, Z.; Fang, W.; Xing, M.; Zhang, J.; J. Solid State Chem. 2015 $226,94$.

65. Abe, R.; Hara, K.; Sayama, K.; Domen, K.; Arakawa, H.; J. Photochem. Photobiol., A 2000, 137, 63.

66. Zheng, H. Q.; Yong, H.; Ou-Yang, T.; Ting Fan, Y.; Hou, H. W.; Int. J. Hydrogen Energy. 2013, 38, 12938.

67. Rao, H.; Yu, W-Q.; Zheng, W-Q.; Bonin, J.; Fan, Y-T.; Hou, H-W.; J. Power Sources 2016, 324, 253.

68. Buchel, G.; Unger, K. K.; Matsumoto, A.; Adv. Mater. 1998, 10,1036.

69. Yamashita, H.; Anpo, M.; Curr. Opin. Solid State Mater. Sci. 2003, 7, 471.

70. Cui, W.; Xu, C.; Zhang, S.; Feng, L.; Lü, S.; Qiu, F.; J. Photochem. Photobiol., A 2005, 175, 89.

71. Liu, S-H.; Paul Wang H.; Int. J. Hydrogen Energy. 2002, 27, 859.

72. Shen, S.; Guo, L.; Catal. Today. 2007, 129, 414.

73. Zhao, D.; Budhi, S.; Rodriguez, A.; Koodali, R. T.; Int. J. Hydrogen Energy. 2010, 35, 5276.

74. Liu, Z.; Shen, S.; Guo, L.; Int. J. Hydrogen Energy. 2012, 37, 816.

75. Ohmori, T.; Mametsuka, H.; Suzuki, E.; Int. J. Hydrogen Energy 2000, 25,953 .

76. Zou, Z.; Ye, J.; Sayama, K.; Arakawa, H.; Nature 2001, 414, 625.

77. Wu, N-L.; Lee, M-S.; Int. J. Hydrogen Energy 2004, 29, 1601.

78. Wang, D.; Zou, Z.; Ye, J.; Chem. Phys. Lett. 2005, 411, 285.

79. Jang, J. S.; Li, W.; Hyuk S.; Lee, J. S.; Chem. Phys. Lett. 2006, 425, 278.

80. Lin, H-Y.; Chang, Y-S.; Int. J. Hydrogen Energy 2010, 35, 8463.

81. Zeng, P.; Zhang, X.; Zhang, X.; Chai, B.; Peng, T.; Chem. L Phys. Lett. 2011, 503, 262.

82. Li, Y.; Zhang, K.; Peng, S.; Lu, G.; Li, S.; J. Mol. Catal. A: Chem. 2012, 363-364, 354.

83. Lyubina, T. P.; Markovskaya, D. V.; Kozlova , E. A.; Parmon, V. N.; Int. J. Hydrogen Energy 2013, 38, 14172.
84. Mou, Z.; Yin, S.; Zhu, M.; Du, Y.; Wang, X.; Yang, P.; Zheng, J.; Lu, C.; Phys. Chem. Chem. Phys. 2013, 15, 2793.

85. Chen, Q.; Suo, C.; Zhang, S.; Wang, Y.; Int. J. Photoenergy 2013, ID 149586, doi: http://dx.doi.org/10.1155/2013/149586

86. Hong, E.; Kim, D.; Kim J. H.; J. Ind. Eng. Chem. 2014, 20, 3869.

87. Du, Q.; Lu, G.; Appl. Surf. Sci. 2014, 305, 235.

88. Zhang, Z.; Cao, S-W.; Liao, Y.; Xue, C.; Appl. Catal., B 2015, 162, 204.

89. Villa, K.; Domenech, X.; García-Pérez, U. M.; Peral, J.; Catal Lett. 2016, 146, 100 .

90. Li, M.; Zhang, L.; Fan, X.; Wu, M.; Du, Y.; Wang, M.; Kong, Q.; Zhang, L.; Shi, J.; Appl. Catal., B 2016, 190, 36.

91. Zhu, Z.; Kao, C-T.; Tang, B-H.; Chang, W-C.; Wu, R-J.; Ceram. Int. 2016, 42, 6749 .

92. Xing, Z.; Li, Z.; Wu, X.; Wang, G.; Zhou, W.; Int. J. Hydrogen Energy 2016, 41, 1535 .

93. Hu, Q.; Huang, J.; Li, G.; Chen, J.; Zhang, Z.; Deng, Z.; Jiang, Y.; Guo, W.; Cao, Y.; Appl. Surf. Sci. 2016, 369, 201.

94. Feil, A. F.; Migowski, P.; Scheffer, F. R.; Pierozan, M. D.; Corsetti, R. R.; Rodrigues, M.; Pezzi, R. P.; Machado, G.; Amaral, L.; Teixeira, S. R.; Weibel, D. E.; Dupont, J.; J. Braz. Chem. Soc. 2010, 21, 1359.

95. Lee, G-J.; Anandan, M. S.; Masten, S. J.; Wu, J. J.; Renewable Energy 2016, 89,18 .

96. Maia, D. L. S.; Pepe, I.; Ferreira da Silva, A.; Silva, L. A.; J. Photochem. Photobiol. A 2012, 243, 61.

97. Nagaraju, G.; Manjunath, K.; Sarkar, S.; Gunter, E.; Teixeira, S. R.; Dupont, J.; Int. J. Hydrogen Energy 2015, 40,12209.

98. Sampaio, M. J.; Oliveira, J. W. L.; Sombrio, C. I. L.; Baptista, D. L.; Teixeira, S. R.; Carabineiro, S. A. C.; Silva, C. G.; Faria, J. L.; Appl. Catal., A 2016, 518, 198.

99. González-Moya, J. R.; Garcia-Basabe, Y.; Rocco, M. L. M.; Pereira, M. B.; Princival, J. L.; Almeida, L. C.; Araújo, C. M.; David, D. G. F.; Silva, A. F.; Machado, G.; Nanotechnology 2016, 27, 285401.

100. Fornari, A. M. D.; Araujo, M. B.; Duarte, C. B.; Machado, G.; Teixeira, S. R.; Weibel, D. E.; J. Hydrogen Energy 2016, 41, 11599.

101. Ortiz, A. L.; Zaragoza, M. M.; Gutíerrez, J. S.; Paula, M. M. S, CollinsMartínez, V.; Int. J. Hydrogen Energy 2015, 40, 17308.

102. Krishnappa, M.; Souza, V. S.; Ganganagappa, N.; Scholten, J. D.; Teixeira, S. R.; Dupont, J.; Thippeswamy R.; Chem. Eur. J. 2015, 21, 17624.

103. Wender, H.; Gonçalves, R. V.; Dias, C. S. B.; Zapata, M. J. M.; Zagonel, L. F.; Mendonça, E. C.; Teixeira, S. R.; Garcia F.; Nanoscale 2013, 5, 9310 . 